\title{
Directing crystallization outcomes of conformationally flexible molecules: polymorphs, solvates, and desolvation pathways of \\ fluconazole
}

Maciej Nowak $^{1}$, Aleksandra J. Dyba ${ }^{1}$, Jan Janczak², Alexander Morritt ${ }^{3}$, László Fábián ${ }^{3}$, Bożena Karolewicz ${ }^{1}$, Yaroslav Z. Khimyak ${ }^{3}$, Doris E. Braun ${ }^{4}$, Karol P. Nartowski ${ }^{1 *}$

${ }^{1}$ Department of Drug Form Technology, Wrocław Medical University, ul. Borowska 211A, 50-556 Wrocław, Poland

${ }^{2}$ Institute of Low Temperature and Structure Research, Polish Academy of Sciences, P.O. Box 1410, Okolna 2 str., 50-950 Wroclaw, Poland

${ }^{3}$ School of Pharmacy, University of East Anglia, Norwich Research Park, NR4 7TJ Norwich, UK

${ }^{4}$ Institute of Pharmacy, University of Innsbruck, Innrain 52c, 6020 Innsbruck, Austria

*Corresponding Author: Karol P. Nartowski; e-mail: karol.nartowski@umed.wroc.pl

Contents of Electronic Supplementary Information:

Contents:

Section S1. Literature and current work nomenclature review and comparison ................................. 2

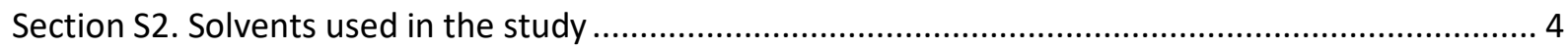

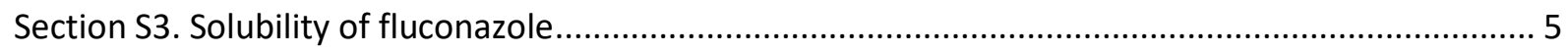

Section S4. Recrystallization of FLU form II from melted sample .................................................... 6

Section S5. PXRD, FTIR, DSC, and TGA analysis of FLU forms I, II, III and IV ......................................... 8

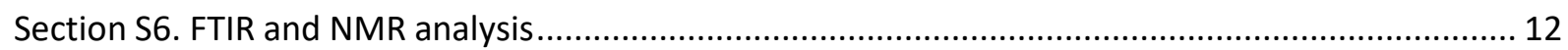

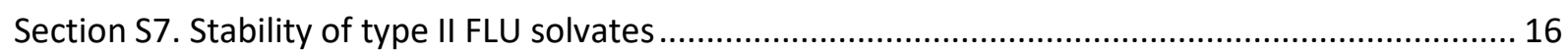

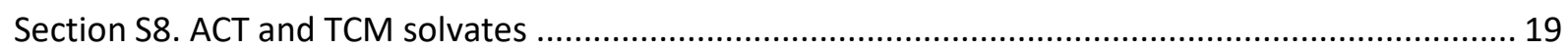

Section S9. Type II fluconazole solvates - structure solution …..................................................... 20

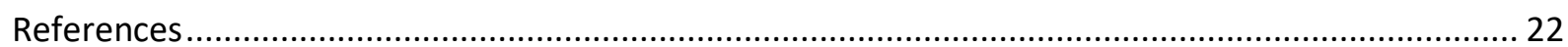




\section{Section S1. Literature and current work nomenclature review and comparison}

Table S1. Literature and current work nomenclature review and comparison.

\begin{tabular}{|c|c|c|c|c|}
\hline Author name & \multicolumn{4}{|c|}{ Reported data (our nomenclature) } \\
\hline Lo at el. ${ }^{1}(1994)$ & $\begin{array}{c}\text { Fluconazole } \\
\text { monohydrate } \\
\text { (mixture of } \\
\text { monohydrate and } \\
\text { polymorph I) (Fig. S1 } \\
\text { left) } \\
\end{array}$ & $\begin{array}{l}\text { Fluconazole } \\
\text { anhydrous } \\
\text { (polymorph III) } \\
\text { (Fig. S1 right) }\end{array}$ & & \\
\hline Gu et al. ${ }^{2}$ (1995) & $\begin{array}{l}\text { Polymorph I } \\
\text { (polymorph I) }\end{array}$ & $\begin{array}{c}\text { Polymorph II } \\
\text { (mixture of } \\
\text { polymorph I and III) }\end{array}$ & & \\
\hline $\begin{array}{l}\text { Dash and Elmquist }{ }^{3} \\
(2001)\end{array}$ & $\begin{array}{l}\text { Polymorph I (mixture } \\
\text { of monohydrate and } \\
\text { polymorph III) }\end{array}$ & $\begin{array}{l}\text { Polymorph II } \\
\text { (polymorph I) }\end{array}$ & $\begin{array}{l}\text { Polymorph III } \\
\text { (polymorph III) }\end{array}$ & $\begin{array}{l}\text { Fluconazole } \\
\text { monohydrate } \\
\text { (Fluconazole } \\
\text { monohydrate) }\end{array}$ \\
\hline Desai et al. ${ }^{4}(2002)$ & $\begin{array}{c}\text { Polymorph I } \\
\text { (polymorph III) }\end{array}$ & $\begin{array}{l}\text { Polymorph II } \\
\text { (polymorph II) }\end{array}$ & & \\
\hline $\begin{array}{l}\text { Alkhamis et al. }{ }^{5} \\
\qquad(2002)\end{array}$ & $\begin{array}{l}\text { Polymorph I } \\
\text { (polymorph I) }\end{array}$ & $\begin{array}{c}\text { Polymorph II } \\
\text { (polymorph II or } \\
\text { product of DCM } \\
\text { solvate desolvation) }\end{array}$ & & \\
\hline Caira et al. ${ }^{6}(2003)$ & $\begin{array}{l}\text { Polymorph III } \\
\text { (polymorph III) } \\
\text { refcode IVUQOF }\end{array}$ & $\begin{array}{c}\text { Fluconazole } \\
\text { monohydrate } \\
\text { (Fluconazole } \\
\text { monohydrate) } \\
\text { refcode IVUQIZ }\end{array}$ & & \\
\hline $\begin{array}{c}\text { Kreidl et al. } 7,8(2002, \\
2006)\end{array}$ & $\begin{array}{l}\text { Crystal modification I } \\
\text { (polymorph I) }\end{array}$ & $\begin{array}{l}\text { Crystal modification II } \\
\text { (polymorph III) }\end{array}$ & & \\
\hline \multirow{3}{*}{$\begin{array}{c}\text { Karanam et al. }{ }^{9} \\
(2012)\end{array}$} & $\begin{array}{l}\text { Polymorph } 1 \text { - } \\
\text { mentioned in } \\
\text { introduction } \\
\text { (polymorph III) }\end{array}$ & $\begin{array}{l}\text { Polymorph 2- } \\
\text { mentioned in } \\
\text { introduction } \\
\text { (polymorph I) }\end{array}$ & $\begin{array}{c}\text { Polymorph } 3- \\
\text { mentioned in } \\
\text { introduction and } \\
\text { obtained by } \\
\text { procedure } 8 \\
\text { described by Kreidl et } \\
\text { al. patent } \\
\text { (polymorph II) }\end{array}$ & $\begin{array}{c}\text { Polymorph } 4 \\
\text { (polymorph IV) } \\
\text { refcode IVUQOF01 }\end{array}$ \\
\hline & $\begin{array}{c}\text { Polymorph } 5 \\
\text { (polymorph I) } \\
\text { refcode IVUQOF02 }\end{array}$ & $\begin{array}{c}\text { Polymorph } 6 \\
\text { (polymorph VI) } \\
\text { refcode IVUQOF03 }\end{array}$ & $\begin{array}{c}\text { Polymorph } 7 \\
\text { (polymorph II) } \\
\text { refcode IVUQOF04 }\end{array}$ & $\begin{array}{c}\text { Polymorph } 8 \\
\text { (mixture of } \\
\text { polymorph I and II) }\end{array}$ \\
\hline & $\begin{array}{l}\text { Polymorph } 9 \\
\text { (mixture of } \\
\text { polymorph I and } \\
\text { monohydrate) }\end{array}$ & & & \\
\hline $\begin{array}{c}\text { Basford et al. }{ }^{10} \\
(2019)\end{array}$ & AH-A (polymorph III) & AH-B (polymorph IV) & $\mathrm{AH}-\mathrm{C}$ (polymorph I) & \\
\hline
\end{tabular}




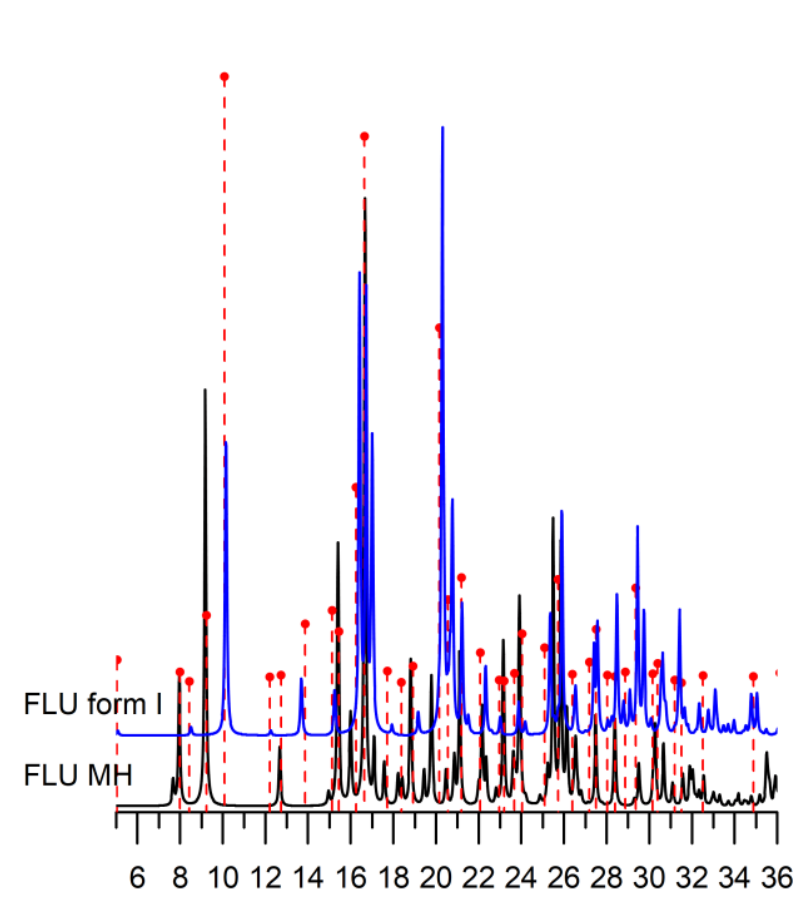

$2 \theta$ (degrees)

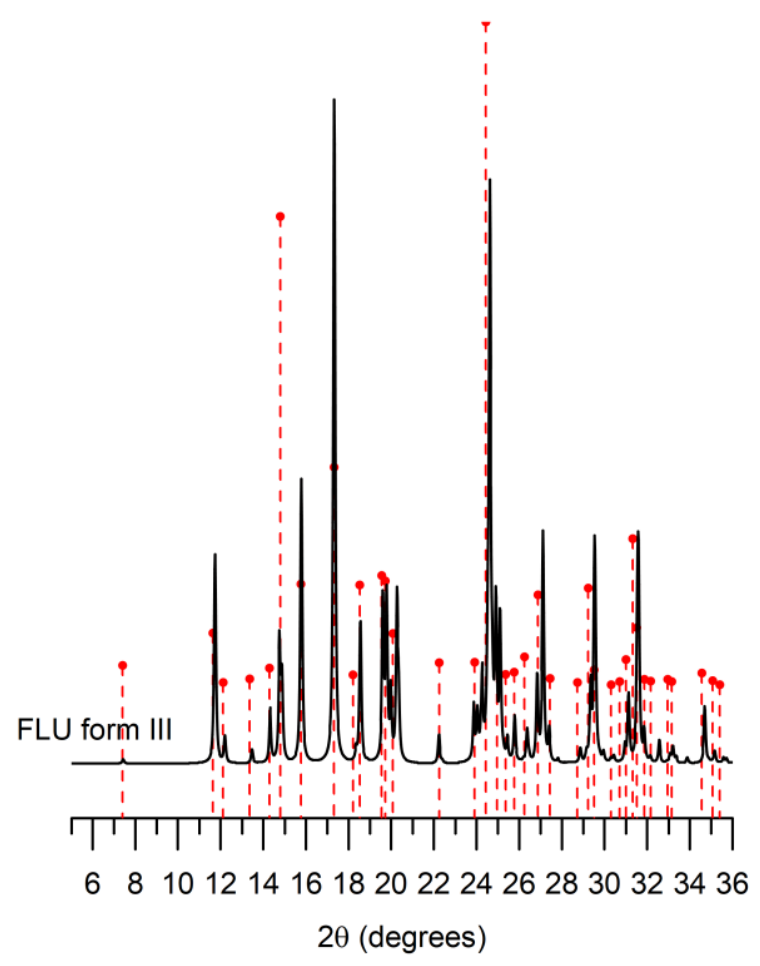

$2 \theta$ (degrees)

Figure S1. Imposition of PXRD data (red dots) of fluconazole monohydrate (left) and fluconazole anhydrous form (right) obtained by Lo et al. ${ }^{1}$ on calculated patterns of fluconazole form I (FLU form I, CSD refcode IVUQOFO2), form III (FLU form III, CSD refcode IVUQOF) and monohydrate (FLU MH, CSD refcode IVUQIZ). 


\section{Section S2. Solvents used in the study}

Table S2. Properties of the solvents used in the study.

\begin{tabular}{|c|c|c|c|c|c|c|c|c|}
\hline Solvent & Formula & $\Sigma \alpha^{a}$ & $\Sigma \beta^{\mathbf{b}}$ & $\begin{array}{l}\text { Dielectric } \\
\text { constant }\end{array}$ & $\begin{array}{c}\text { Dipole } \\
\text { momentum }\end{array}$ & $\delta D^{d}$ & $\delta P^{d}$ & $\delta H^{d}$ \\
\hline Water $\left(\mathrm{H}_{2} \mathrm{O}\right)$ & $\mathrm{H}_{2} \mathrm{O}$ & 1.17 & 0.47 & 78.36 & 1.87 & 15.5 & 16.0 & 42.3 \\
\hline Methanol (MeOH) & $\mathrm{CH}_{3} \mathrm{OH}$ & 0.43 & 0.47 & 32.61 & 1.70 & 14.7 & 12.3 & 22.3 \\
\hline Ethanol (EtOH) & $\mathrm{C}_{2} \mathrm{H}_{5} \mathrm{OH}$ & 0.37 & 0.48 & 24.85 & 1.69 & 15.8 & 8.8 & 19.4 \\
\hline n-Propanol (nPrOH) & $\mathrm{C}_{3} \mathrm{H}_{7} \mathrm{OH}$ & 0.37 & 0.48 & 20.52 & 1.55 & 16.0 & 6.8 & 17.4 \\
\hline $\begin{array}{l}\text { Iso-propanol } \\
\text { (isPrOH) }\end{array}$ & $\mathrm{C}_{3} \mathrm{H}_{7} \mathrm{OH}$ & 0.33 & 0.56 & 19.26 & 1.56 & 15.8 & 6.1 & 16.4 \\
\hline n-Butanol (BuOH) & $\mathrm{C}_{4} \mathrm{H}_{9} \mathrm{OH}$ & 0.37 & 0.48 & 17.33 & 1.66 & 16.0 & 5.7 & 15.8 \\
\hline Butanone (BuON) & $\mathrm{C}_{4} \mathrm{H}_{8} \mathrm{O}$ & 0.00 & 0.51 & 18.25 & 2.78 & 16.0 & 9.0 & 5.1 \\
\hline Acetone (ACT) & $\mathrm{CH}_{3} \mathrm{COCH}_{3}$ & 0.04 & 0.49 & 20.49 & 2.88 & 15.5 & 10.4 & 7.0 \\
\hline Acetonitrile (ACN) & $\mathrm{CH}_{3} \mathrm{CN}$ & 0.07 & 0.32 & 35.69 & 3.92 & 15.3 & 18.0 & 6.1 \\
\hline $\begin{array}{l}\text { Dimethylformamide } \\
\text { (DMF) }\end{array}$ & $\left(\mathrm{CH}_{3}\right)_{2} \mathrm{NCHO}$ & 0.00 & 0.74 & 37.22 & 3.82 & 17.4 & 13.7 & 11.3 \\
\hline $\begin{array}{l}\text { Dimethyl sulfoxide } \\
\text { (DMSO) }\end{array}$ & $\mathrm{CH}_{3} \mathrm{SOCH}_{3}$ & 0.00 & 0.88 & 46.83 & 1.80 & 18.4 & 16.4 & 10.2 \\
\hline Ethyl acetate (EtAc) & $\mathrm{CH}_{3} \mathrm{COOC}_{2} \mathrm{H}_{5}$ & 0.00 & 0.45 & 5.99 & 1.78 & 15.8 & 5.3 & 7.2 \\
\hline Toluene (Tol) & $\mathrm{C}_{6} \mathrm{H}_{5} \mathrm{CH}_{3}$ & 0.00 & 0.14 & 2.37 & 0.38 & 18.0 & 1.4 & 2.0 \\
\hline $\begin{array}{l}\text { Dichloromethane } \\
\text { (DCM) }\end{array}$ & $\mathrm{CH}_{2} \mathrm{Cl}_{2}$ & 0.10 & 0.05 & 8.93 & 1.60 & 17.0 & 7.3 & 7.1 \\
\hline Chloroform (TCM) & $\mathrm{CHCl}_{3}$ & 0.15 & 0.02 & 4.71 & 1.04 & 17.8 & 3.1 & 5.7 \\
\hline \multicolumn{9}{|c|}{$\begin{array}{l}\left.\text { a - Abraham's } s^{11} \text { hydrogen bond acidity (Abraham's notation } \Sigma \alpha_{2}{ }^{H}\right),{ }^{b}-\text { Abraham's }{ }^{11} \text { hydrogen bond basicity (Abraham's } \\
\left.\text { notation } \Sigma \beta_{2}{ }^{H}\right), c-\text { Dipole moment in Debye; d - Hansen solubility parameter values accounting for dispersion forces } \\
(\delta D) \text {, dipolar intermolecular forces }(\delta P) \text {, and hydrogen bonds between molecules }(\delta H) \text { in } M \mathrm{MPa}^{0.5}\end{array}$} \\
\hline
\end{tabular}




\section{Section S3. Solubility of fluconazole}

Table S3. Solubility of fluconazole polymorphic forms and solvates in organic solvents at room temperature $\left(22-24{ }^{\circ} \mathrm{C}\right)$ and at ice bath temperature.

\begin{tabular}{|c|c|c|c|c|c|c|c|c|c|c|c|c|c|c|}
\hline $\begin{array}{c}\text { Solubility } \\
\text { (mg/mL) }\end{array}$ & $\mathrm{MeOH}$ & SD & EtOH & SD & IsPrOH & SD & DMF & SD & $\mathrm{nPrOH}$ & SD & $\mathrm{nBuOH}$ & SD & DMSO & SD \\
\hline FI & $\begin{array}{c}361.7 \\
\text { Ice } \\
\text { bath: } \\
184.9\end{array}$ & 15.3 & $\begin{array}{c}100.3 \\
\text { Ice } \\
\text { bath: } \\
48.6\end{array}$ & 5.9 & $\begin{array}{c}39.2 \\
\text { Ice } \\
\text { bath: } \\
17.0\end{array}$ & 5.7 & $\begin{array}{l}554.9 \\
\text { Ice } \\
\text { bath: } \\
242.0\end{array}$ & 19.3 & 60.9 & 2.0 & 41.2 & 4.0 & 678.4 & 8.5 \\
\hline FII & 321.6 & 5.5 & 78.6 & 6.3 & 30.0 & 2.2 & 553.1 & 5.0 & & & & & & \\
\hline FIII & 302.1 & 9.1 & 79.8 & 3.9 & 30.9 & 3.0 & 500.6 & 9.9 & & & & & & \\
\hline FIV & 283.6 & 34.3 & 78.8 & 10.4 & 38.8 & 0.4 & 436.6 & 0.3 & & & & & 351.5 & 7.8 \\
\hline STI & & & & & & & & & & & 37.9 & 0.2 & 548.3 & 32.6 \\
\hline STII & & & & & & & & & 41.5 & 5.7 & 22.4 & 2.1 & & \\
\hline $\begin{array}{l}\text { Solubility } \\
\text { (mg/mL) }\end{array}$ & ACN & SD & DCM & SD & TCM & SD & ACT & SD & BuON & SD & TOL & SD & EtAc & SD \\
\hline FI & 63.9 & 4.3 & & & Immedia & tran & on to $\mathrm{FL}$ & solvate & & & $\begin{array}{r}\text { Undete } \\
\text { by H }\end{array}$ & $\begin{array}{l}\text { able } \\
\text { C }\end{array}$ & 26.9 & 0.9 \\
\hline \multicolumn{15}{|l|}{ FII } \\
\hline FIII & & & & & & & & & 45.25 & 3.0 & & & & \\
\hline FIV & & & & & & & & & 40.5 & 4.0 & & & & \\
\hline STI & 56.4 & 6.5 & & & & & & & 47.3 & 0.8 & \multicolumn{2}{|c|}{$\begin{array}{c}\text { Undetectable } \\
\text { by HPLC }\end{array}$} & 22.9 & 2.3 \\
\hline STII & 39.3 & 3.3 & 43.4 & 1.8 & 52.4 & 6.5 & 55.2 & 3.3 & & & & & & \\
\hline
\end{tabular}

SD - standard deviation.

Table S4. Fluconazole solubility in solvent-water mixtures. Water activity $\left(a_{w}\right)$ was calculated based on ${ }^{12-15}$.

\begin{tabular}{|c|c|c|c|}
\hline Solubility $(\mathrm{mg} / \mathrm{mL})$ & Water 5\% (v/v) & Water $10 \%(v / v)$ & Water $20 \%(v / v)$ \\
\hline $\mathrm{MeOH}$ & $322.7 \pm 12.7 \quad\left(a_{w}=0.12\right)$ & $343.7 \pm 10.5\left(a_{w}=0.21\right)$ & $\begin{array}{c}284.2 \pm 2.9\left(\mathrm{a}_{\mathrm{w}}=0.37\right) \\
\text { Hydrate formation }\end{array}$ \\
\hline EtOH & $156.4 \pm 14.2\left(\mathrm{a}_{\mathrm{w}}=0.33\right)$ & $163.19 \pm 16.77\left(\mathrm{a}_{\mathrm{w}}=0.52\right)$ & $\begin{array}{c}155.4 \pm 35.4\left(\mathrm{a}_{\mathrm{w}}=0.70\right) \\
\text { Hydrate formation }\end{array}$ \\
\hline IsPrOH & $\begin{array}{l}75.0 \pm 8.0\left(a_{w}=0.46\right) \\
\text { Hydrate formation }\end{array}$ & $\begin{array}{l}82.28 \pm 5.3\left(a_{w}=0.68\right) \\
\text { Hydrate formation }\end{array}$ & $\begin{array}{c}109.9 \pm 5.6\left(a_{w}=0.83\right) \\
\text { Hydrate formation }\end{array}$ \\
\hline DMF & $524.3 \pm 21.1\left(\mathrm{a}_{\mathrm{w}}=0.24\right)$ & $484.7 \pm 8.6\left(a_{w}=0.38\right)$ & $\begin{array}{c}369.2 \pm 9.64\left(\mathrm{a}_{\mathrm{w}}=0.56\right) \\
\text { Hydrate formation }\end{array}$ \\
\hline
\end{tabular}


Section S4. Recrystallization of FLU form II from melted sample

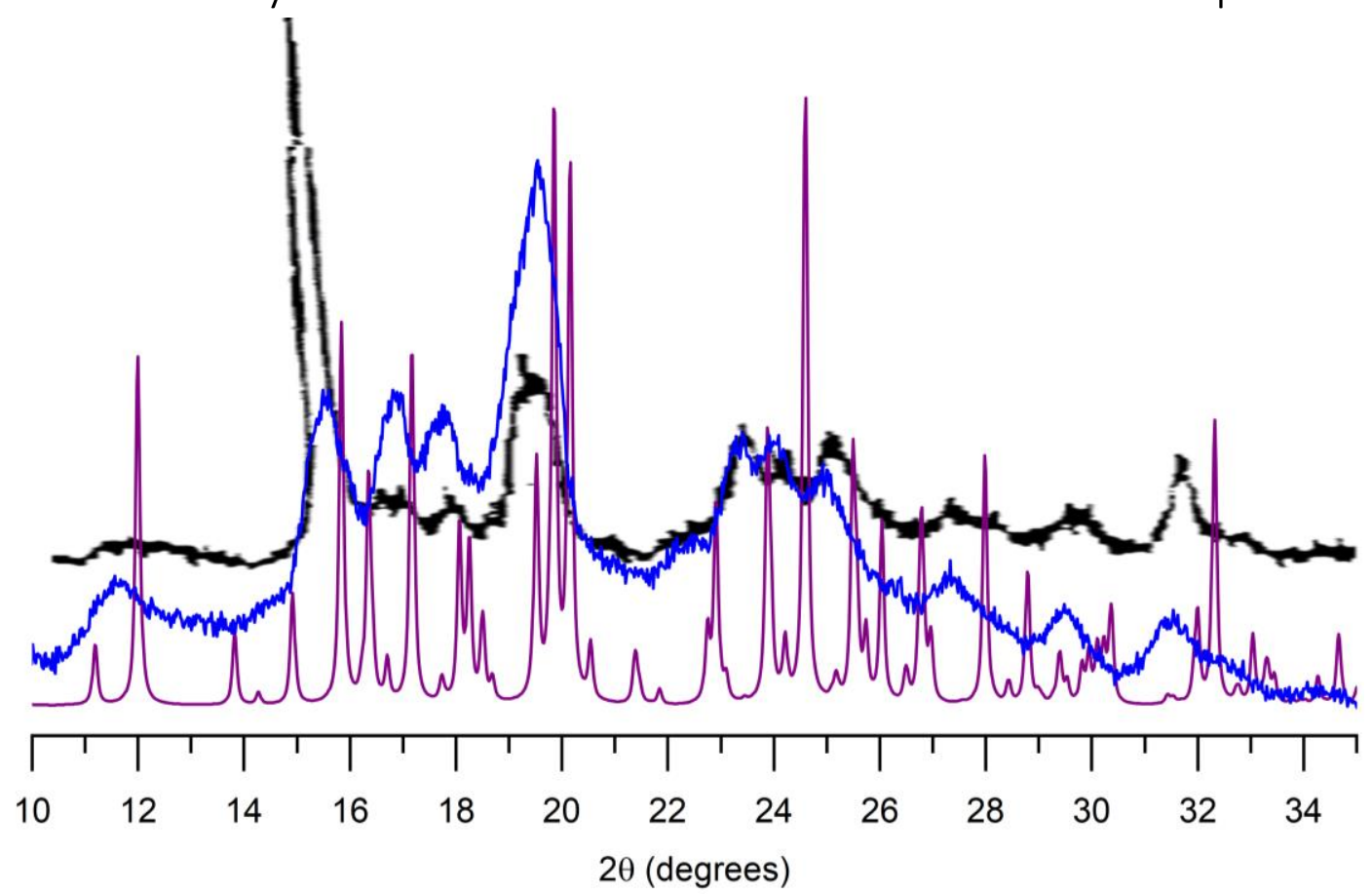

Figure S2. Experimental PXRD patterns of FLU form II obtained by crystallization from melted fluconazole (black - Desai et al. ${ }^{4}$, blue - our data) superimposed on calculated pattern of fluconazole form II at 100K (purple, CSD ref. code IVUQOFO4).

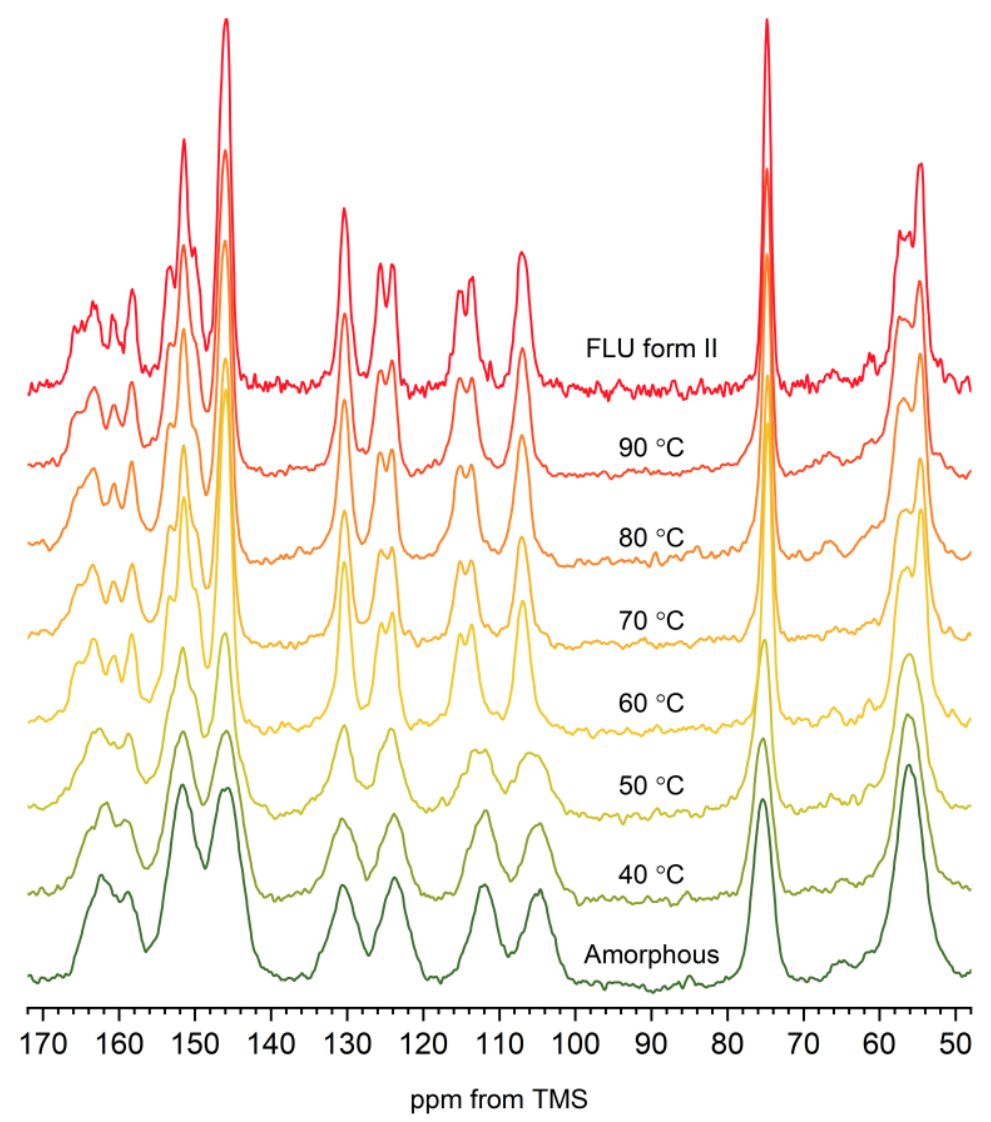

Figure S3. Variable temperature solid state NMR spectra of fluconazole form II (FLU form II) crystallized from melt. 


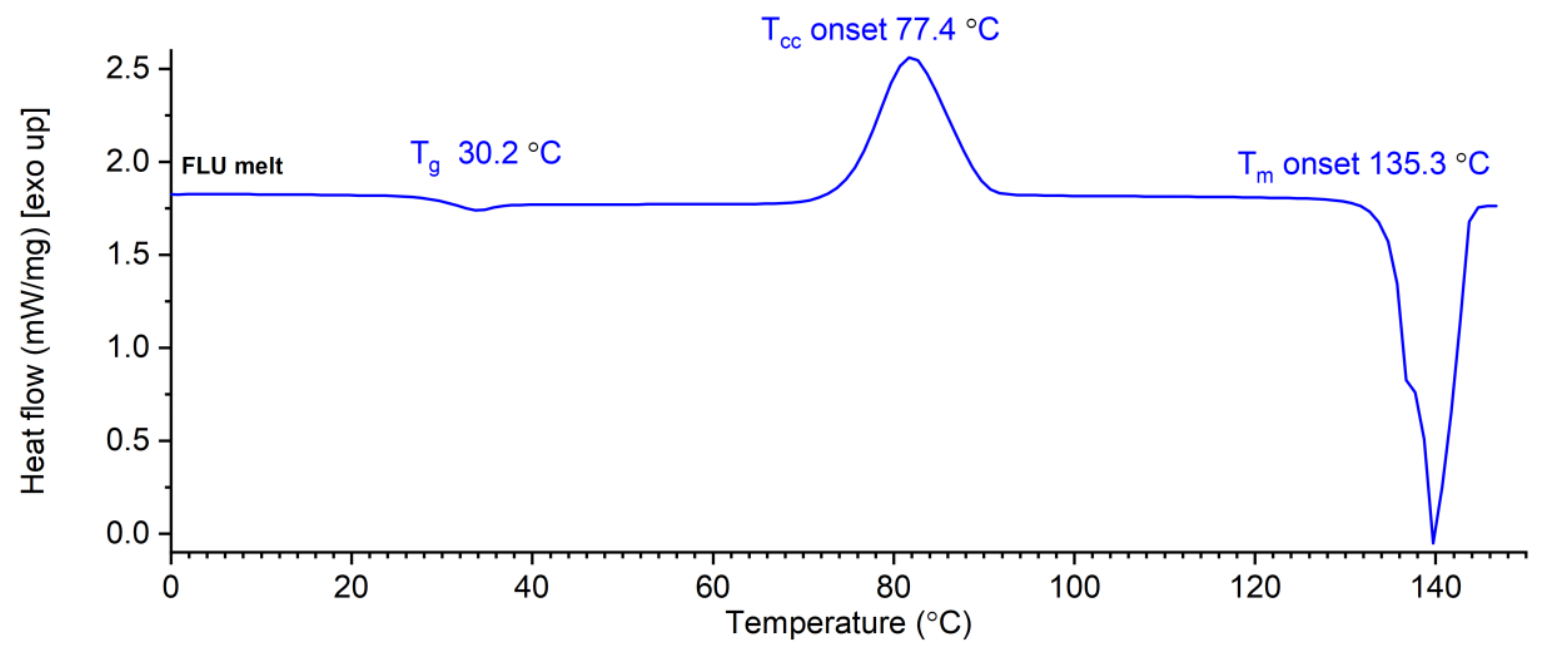

Figure S4. DSC thermogram of melted fluconazole. 
Section S5. PXRD, FTIR, DSC, and TGA analysis of FLU forms I, II, III and IV
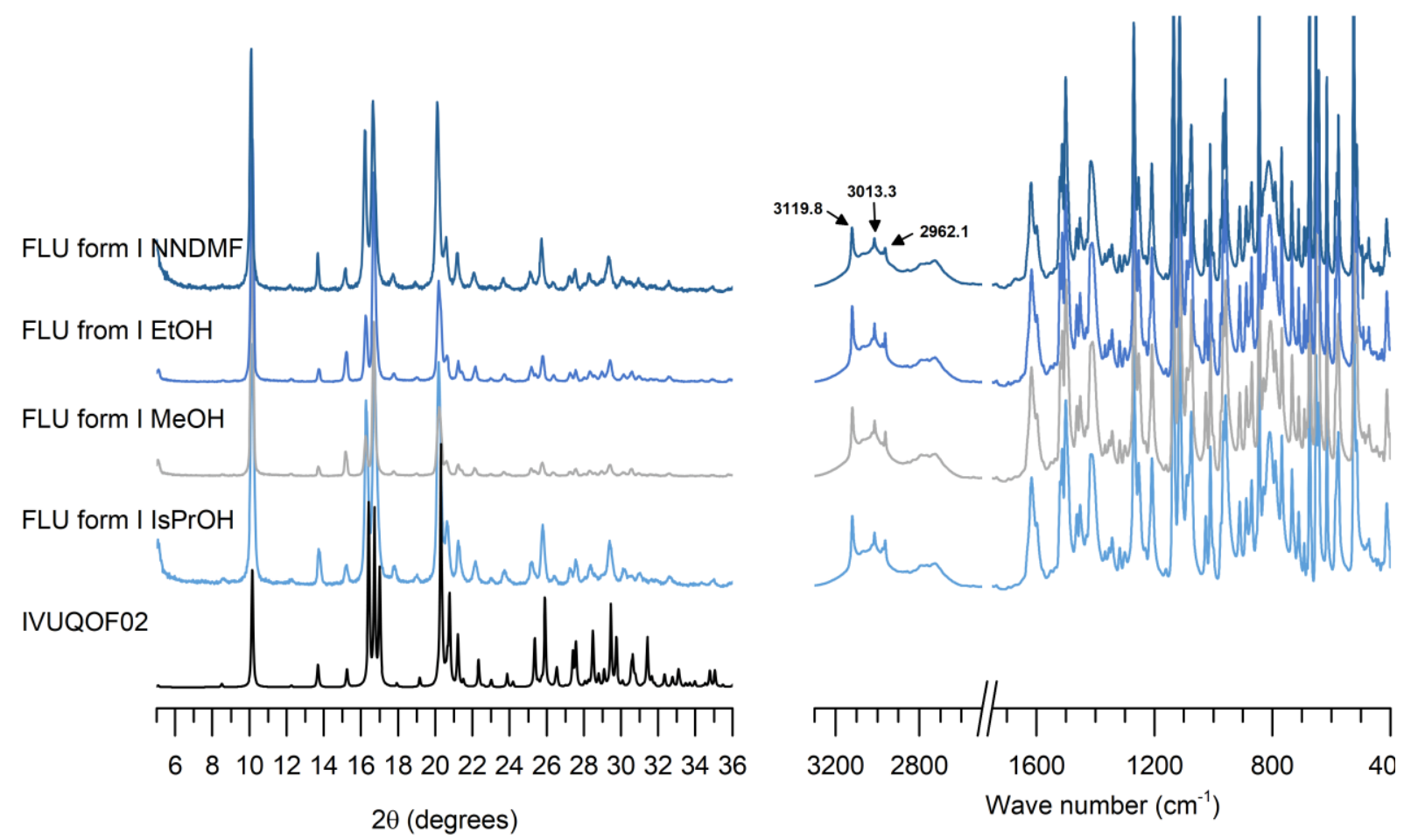

Figure S5. Experimental and calculated (CSD refcode IVUQOFO2) PXRD patterns (left) and experimental FTIR spectra (right) of fluconazole form I (FLU form I) obtained via cooling crystallization in isopropanol (IsPrOH), methanol (MeOH), ethanol (EtOH) and N,N-dimethylformamide (NNDMF).
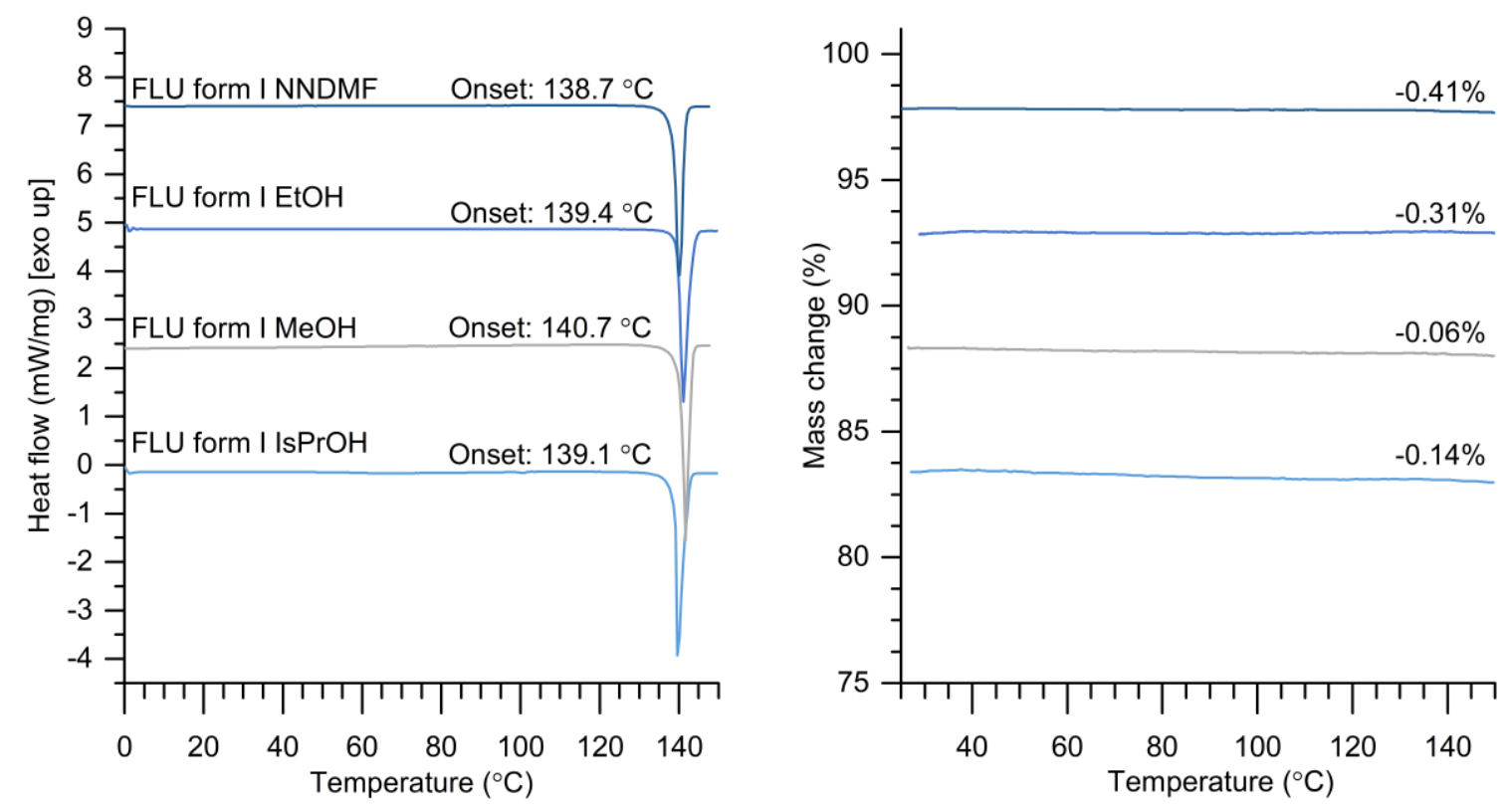

Figure S6. DSC (left) and TGA (right) thermograms of fluconazole form I (FLU form I) obtained via cooling crystallization in isopropanol (IsPrOH), methanol (MeOH), ethanol (EtOH) and N,N-dimethylformamide (NNDMF). 

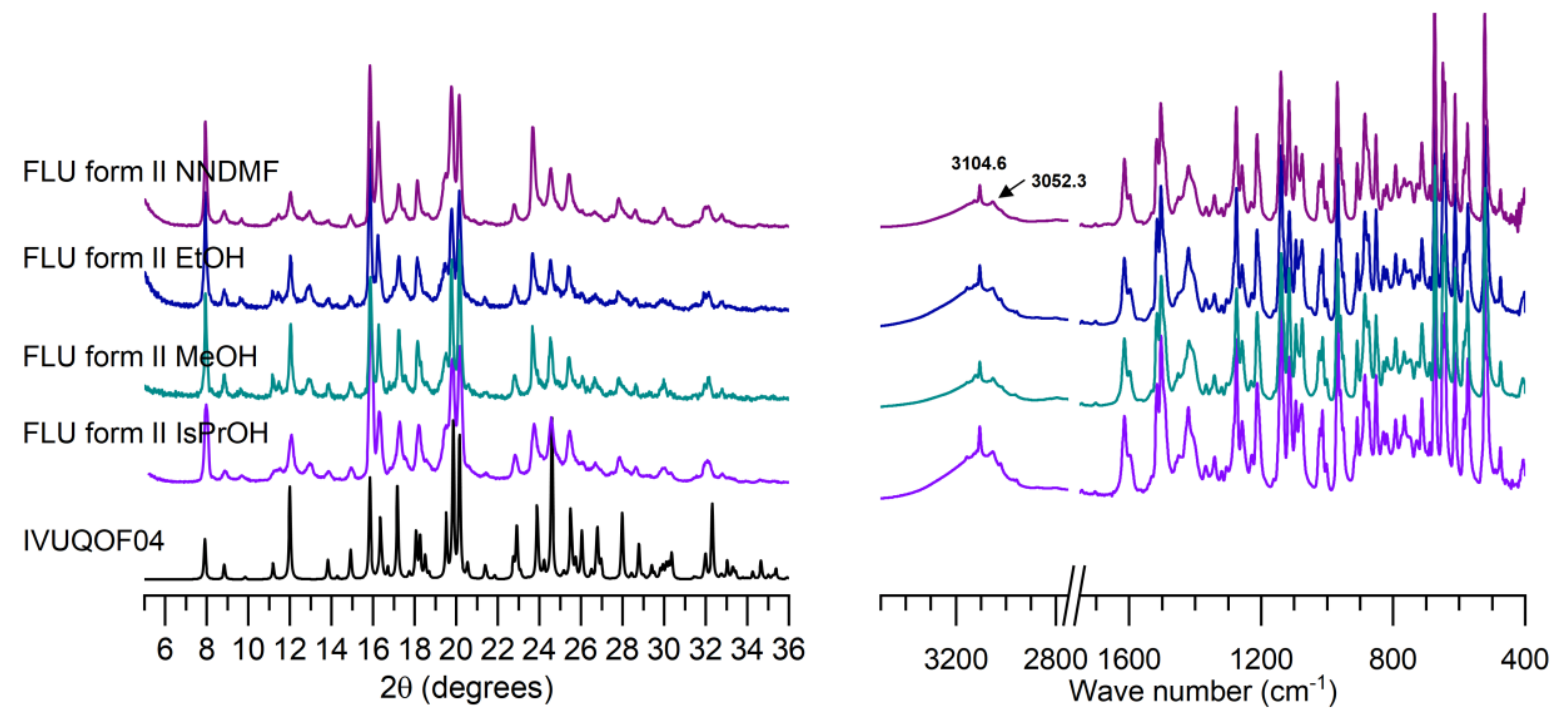

Figure S7. Experimental and calculated (CSD refocde IVUQOFO4) PXRD patterns (left) and experimental FTIR spectra (right) of fluconazole form II (FLU form II) obtained via cooling crystallization in isopropanol (IsPrOH), methanol (MeOH), ethanol (EtOH) and N,N-dimethylformamide (NNDMF).
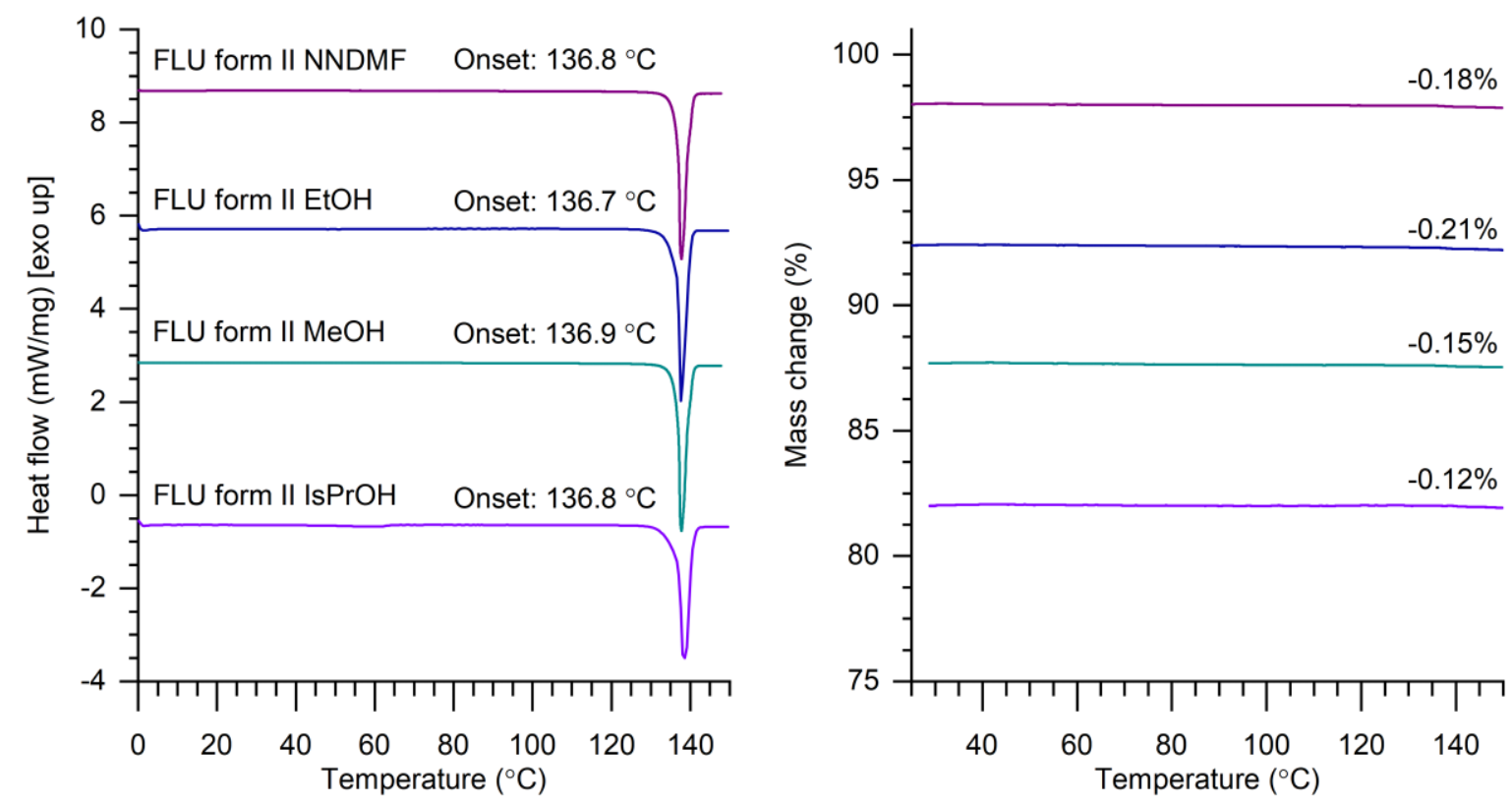

Figure S8. DSC (left) and TGA (right) thermograms of fluconazole form II (FLU form II) obtained via cooling crystallization in isopropanol (IsPrOH), methanol (MeOH), ethanol (EtOH) and N,N-dimethylformamide (NNDMF). 

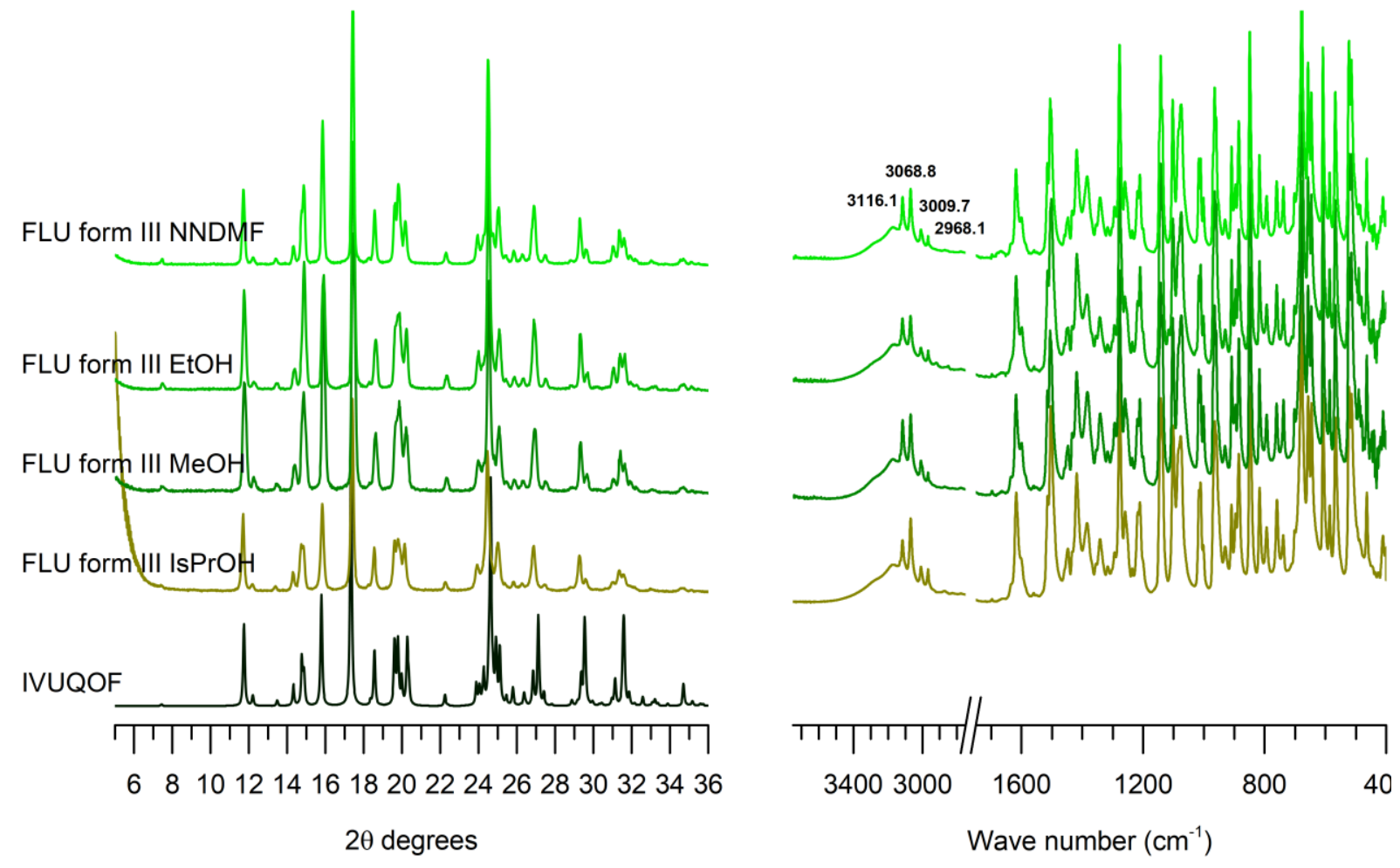

Figure S9. Experimental and calculated (CSD refcode IVUQOF) PXRD patterns (left) and experimental FTIR spectra (right) of fluconazole form III (FLU form III) obtained via slurring crystallization in isopropanol (IsPrOH), methanol (MeOH), ethanol (EtOH) and N,N-dimethylformamide (NNDMF).
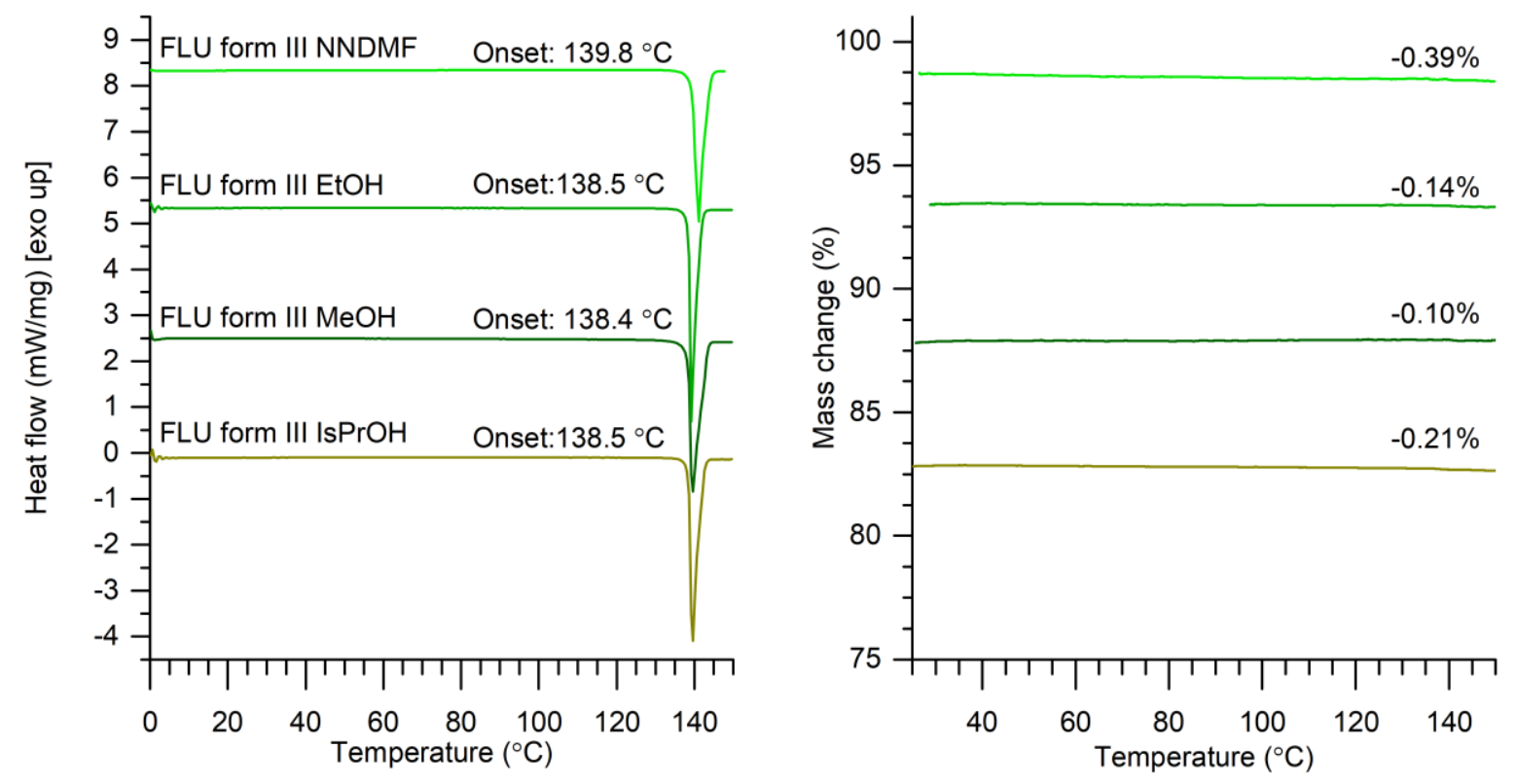

Figure S10. DSC (left) and TGA (right) thermograms of fluconazole form III (FLU form IIII) obtained via slurring crystallization in isopropanol (IsPrOH), methanol (MeOH), ethanol (EtOH) and $\mathrm{N}, \mathrm{N}$-dimethylformamide (NNDMF). 

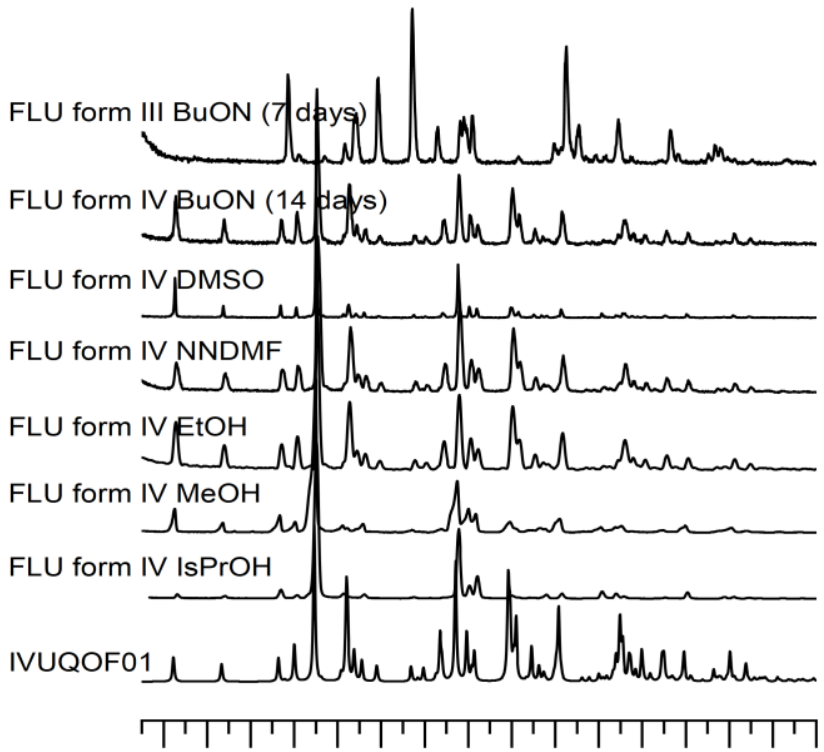

681012141618202224262830323436 $2 \theta$ (degrees)
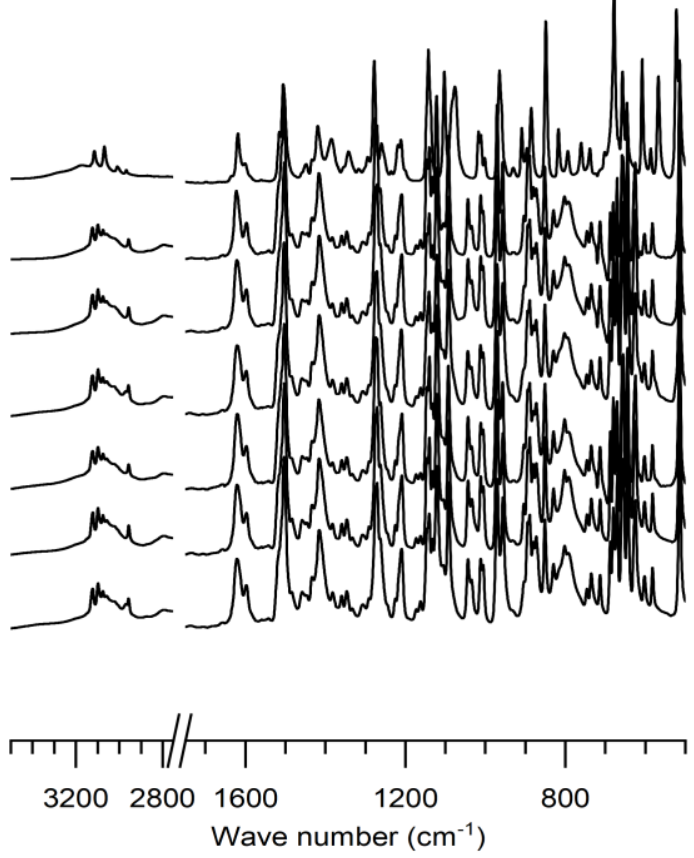

Figure S11. Experimental and calculated (CSD refcode IVUQOFO1) PXRD patterns (left) and experimental FTIR spectra (right) of fluconazole form IV (FLU form IV) obtained via slurring crystallization in isopropanol (IsPrOH), methanol (MeOH), ethanol $(E t O H), N N$-dimethylformamide (NNDMF), dimethyl sulfoxide (DMSO) and butanone (BuON).
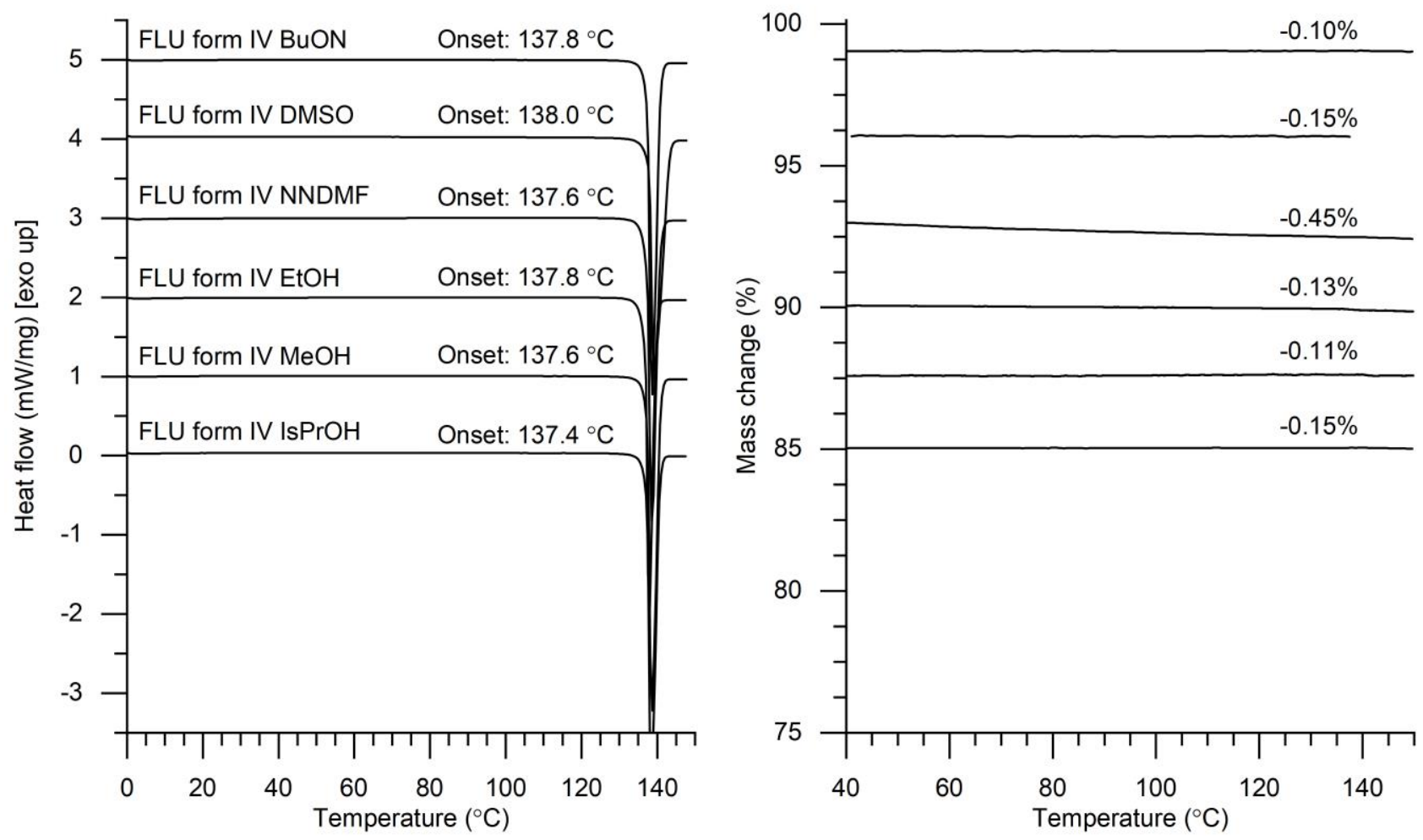

Figure S12. DSC (left) and TGA (right) thermograms of fluconazole form IV (FLU form IV) obtained via slurring crystallization in isopropanol (IsPrOH), methanol (MeOH), ethanol (EtOH), NN-dimethylformamide (NNDMF), dimethyl sulfoxide (DMSO) and butanone (BUON). 
Section S6. FTIR and NMR analysis

Table S5. FTIR vibrational bands assignments of FLU polymorphs and solvates.

\begin{tabular}{|c|c|c|c|c|c|c|c|}
\hline $\begin{array}{l}\text { Vibrational band } \\
\text { assignments }\end{array}$ & FLU form I & FLU form II & FLU form III & $\begin{array}{l}\text { FLU form } \\
\text { IV }\end{array}$ & $\begin{array}{c}\text { FLU } \\
\text { hydrate }\end{array}$ & $\begin{array}{l}\text { FLU type I } \\
\text { solvates }\end{array}$ & $\begin{array}{l}\text { FLU type II } \\
\text { solvates }\end{array}$ \\
\hline Triazole C-H stretching & 3019.8 & $\begin{array}{l}3126.0 \\
3104.3\end{array}$ & 3115.9 & $\begin{array}{l}3124.12 \\
3099.53\end{array}$ & $\begin{array}{l}3115.4 \\
3106.3\end{array}$ & 3119.3 & $\begin{array}{l}3017.8 \\
3105.3\end{array}$ \\
\hline $\begin{array}{l}\text { Difluorophenyl CH } \\
\text { stretching }\end{array}$ & 3013.2 & 3051.8 & $\begin{array}{l}3068.7 \\
3009.9\end{array}$ & 3075.42 & $\begin{array}{l}3060.9 \\
3020.0\end{array}$ & 3013.2 & $\begin{array}{l}3073.9 \\
3041.6\end{array}$ \\
\hline $\begin{array}{l}\text { Methylene C-H asymmetric } \\
\text { stretching }\end{array}$ & 2962.12 & & 2967.9 & 2957.8 & 2956.8 & 2962.1 & 2963.5 \\
\hline $\begin{array}{c}\text { Difluorophenyl ring } \mathrm{C}=\mathrm{C} \\
\text { stretching }\end{array}$ & 1617.0 & 1614.5 & 1618.5 & 1622.8 & 1618.0 & 1615.5 & 1615.1 \\
\hline $\begin{array}{c}\text { Difluorophenyl C=C } \\
\text { stretching }\end{array}$ & 1598.7 & 1598.2 & 1601.1 & 1597.25 & 1591.0 & 1598.2 & 1598.2 \\
\hline Triazole $\mathrm{C}=\mathrm{N}$ stretching & $\begin{array}{l}1519.6 \\
1512.8 \\
1500.3\end{array}$ & $\begin{array}{l}1516.7 \\
1503.7\end{array}$ & $\begin{array}{l}1515.3 \\
1504.6\end{array}$ & 1503.7 & $\begin{array}{l}1514.8 \\
1504.7\end{array}$ & $\begin{array}{l}1519.1 \\
1512.9 \\
1498.9\end{array}$ & $\begin{array}{l}1519.1 \\
1501.8\end{array}$ \\
\hline $\begin{array}{c}\text { Difluorophenyl ring C-C } \\
\text { stretching }\end{array}$ & 1463.2 & & & & 1467.1 & 1463.2 & 1460.8 \\
\hline Methylene scissoring & 1451.6 & 1450.7 & 1448.3 & 1446.3 & 1445.4 & 1451.6 & 1444.9 \\
\hline $\begin{array}{l}\text { Methylene scissoring or } \\
\text { triazole C-N stretching }\end{array}$ & 1410.2 & 1420.3 & 1418.4 & 1415.9 & 1420.3 & 1410.1 & 1417.9 \\
\hline Methylene wagging & $\begin{array}{l}1367.3 \\
1353.3\end{array}$ & 1367.3 & 1384.16 & $\begin{array}{l}1382.7 \\
1359.1\end{array}$ & $\begin{array}{l}1370.6 \\
1358.6\end{array}$ & $\begin{array}{l}1367.7 \\
1353.3\end{array}$ & $\begin{array}{l}1366.3 \\
1355.7\end{array}$ \\
\hline Triazole C-N stretching & $\begin{array}{l}1343.2 \\
1317.6 \\
1300.2\end{array}$ & $\begin{array}{l}1341.7 \\
1321.0 \\
1304.6\end{array}$ & $\begin{array}{l}1342.2 \\
1316.6 \\
1294.5\end{array}$ & $\begin{array}{l}1346.5 \\
1333.5 \\
1306.5\end{array}$ & $\begin{array}{l}1348.0 \\
1317.6 \\
1305.1 \\
\end{array}$ & $\begin{array}{l}1342.7 \\
1316.7 \\
1300.7\end{array}$ & $\begin{array}{l}1345.6 \\
1327.7\end{array}$ \\
\hline $\begin{array}{l}\text { O-H in-plane-bending (C-OH } \\
\text { scissoring) }\end{array}$ & 1269.4 & 1275.2 & 1277.6 & 1274.7 & 1274.7 & 1269.4 & 1274.2 \\
\hline C-F stretching & 1253.0 & 1258.3 & 1260.2 & 1262.6 & 1248.2 & 1252.5 & 1255.9 \\
\hline $\begin{array}{c}\text { C-C stretching, } \\
\text { difluorophenyl in plane } \\
\text { banding }(\mathrm{H}-\mathrm{C}=\mathrm{C})\end{array}$ & 1230.8 & 1230.8 & 1235.6 & 1225.0 & 1216.4 & 1234.7 & \\
\hline $\begin{array}{l}\text { Triazole N-N stretching, } \\
\text { Methylene twisting }\end{array}$ & 1208.2 & 1212.5 & $\begin{array}{l}1218.3 \\
1210.6\end{array}$ & 1209.1 & 1208.18 & 1208.2 & 1211.1 \\
\hline $\begin{array}{l}\text { Difluorophenyl ring } \mathrm{C}-\mathrm{H} \text { - in- } \\
\text { plane bending }\end{array}$ & $\begin{array}{l}1133.9 \\
1113.7\end{array}$ & $\begin{array}{l}1140.2 \\
1131.5 \\
1115.6\end{array}$ & $\begin{array}{l}1142.6 \\
1102.1\end{array}$ & $\begin{array}{l}1146.5 \\
1140.2 \\
1132.5\end{array}$ & $\begin{array}{l}1137.8 \\
1110.8\end{array}$ & $\begin{array}{l}1134.4 \\
1114.6\end{array}$ & $\begin{array}{l}1139.7 \\
1130.1 \\
1119.9\end{array}$ \\
\hline $\begin{array}{l}\text { C-O-H bending, } \mathrm{C}-\mathrm{C}-\mathrm{C} \\
\text { trigonal bending }\end{array}$ & 1025.9 & 1022.0 & 1016.8 & 1043.3 & 1020.1 & 1025.4 & 1025.9 \\
\hline Triazole $\mathrm{N}=\mathrm{C}-\mathrm{N}$ bending & 1010.0 & 1014.9 & 1011.0 & 1011.9 & 1015.3 & 1010.0 & 1010.5 \\
\hline $\begin{array}{l}\text { Triazole N-N stretching, } \\
\text { methylene rocking }\end{array}$ & 968.0 & 969.2 & 964.2 & 970.5 & 965.7 & 965.7 & 969.1 \\
\hline $\mathrm{C}-\mathrm{C}-\mathrm{C}$ ring breathing & 959.9 & 958.9 & & 957.0 & & 958.0 & 960.4 \\
\hline $\begin{array}{l}\text { Triazole } \mathrm{C}-\mathrm{N}=\mathrm{C} \text { scissoring, } \\
\text { difluorophenyl out-of-plane- } \\
\text { bending }\end{array}$ & 910.2 & 909.3 & 908.8 & 903.5 & 916.5 & 910.2 & 906.4 \\
\hline
\end{tabular}




\begin{tabular}{|c|c|c|c|c|c|c|c|}
\hline $\begin{array}{l}\text { C-O bending, triazole in- } \\
\text { plane-bending, methylene } \\
\text { rocking }\end{array}$ & 888.0 & 885.7 & 885.1 & $\begin{array}{l}894.3 \\
889.5\end{array}$ & 898.18 & 887.6 & 885.1 \\
\hline $\begin{array}{l}\text { Triazole ring out-of-plane- } \\
\text { bending }\end{array}$ & 869.3 & 875.0 & & $\begin{array}{l}880.3 \\
873.1\end{array}$ & 874.6 & 869.3 & 875.5 \\
\hline $\begin{array}{l}\text { Difluorophenyl C-H out-of- } \\
\text { plane-bending }\end{array}$ & 845.15 & 851.9 & 848.5 & 851.4 & 852.4 & $\begin{array}{l}853.4 \\
845.6\end{array}$ & \\
\hline $\begin{array}{l}\text { Triazole ring out-of-plane- } \\
\text { bending }\end{array}$ & 829.7 & $\begin{array}{l}829.7 \\
819.6\end{array}$ & 816.7 & 829.7 & 833.1 & 828.8 & 829.7 \\
\hline $\begin{array}{l}\text { Difluorophenyl C-H out-of- } \\
\text { plane-bending }\end{array}$ & $\begin{array}{l}806.6 \\
789.2\end{array}$ & 792.6 & 794.0 & $\begin{array}{l}802.2 \\
792.1\end{array}$ & & $\begin{array}{l}802.7 \\
789.2\end{array}$ & 798.4 \\
\hline $\begin{array}{l}\text { C-N asymmetric stretching } \\
\text { (skeleton), C-C asymmetric } \\
\text { stretching (skeleton), } \\
\text { triazole bending N-C-C }\end{array}$ & 768.0 & 766.0 & 760.3 & & 766.1 & 768.1 & \\
\hline $\begin{array}{l}\text { C-N symmetric stretching } \\
\text { (skeleton), triazole bending } \\
(\mathrm{N}=\mathrm{C}-\mathrm{N})\end{array}$ & 732.8 & 728.5 & 738.1 & 735.2 & 734.3 & 732.3 & \\
\hline C-C-C in-plane-bending & $\begin{array}{l}710.6 \\
691.4 \\
673.0\end{array}$ & $\begin{array}{l}713.0 \\
688.9 \\
674.0\end{array}$ & $\begin{array}{c}701.5 \\
677.8\end{array}$ & $\begin{array}{l}713.0 \\
687.9 \\
680.7 \\
670.6\end{array}$ & $\begin{array}{l}679.3 \\
673.0\end{array}$ & $\begin{array}{l}710.6 \\
691.8 \\
673.5\end{array}$ & $\begin{array}{l}713.5 \\
674.5\end{array}$ \\
\hline $\begin{array}{l}\text { Triazole ring out-of-plane- } \\
\text { bending }\end{array}$ & $\begin{array}{l}651.3 \\
642.2 \\
614.7\end{array}$ & $\begin{array}{l}649.4 \\
643.1 \\
612.8\end{array}$ & $\begin{array}{l}657.1 \\
646.0 \\
607.4\end{array}$ & $\begin{array}{l}658.1 \\
644.5 \\
625.3\end{array}$ & $\begin{array}{l}652.3 \\
616.6\end{array}$ & $\begin{array}{l}651.3 \\
641.7 \\
614.7\end{array}$ & $\begin{array}{l}652.3 \\
617.6\end{array}$ \\
\hline C-C-C in-plane-bending & 585.3 & 586.7 & 586.7 & 582.4 & 587.2 & & 588.7 \\
\hline C-F in-plane-bending & 575.7 & 573.7 & 567.0 & & 569.9 & 575.7 & 575.2 \\
\hline $\mathrm{N}-\mathrm{C}-\mathrm{C}$ bending & 523.1 & 522.1 & 521.7 & & 530.3 & 523.6 & 523.6 \\
\hline C-C-C out-of-plane-bending & 513.5 & 515.9 & 514.9 & 514.4 & 513.5 & 513.4 & \\
\hline Solvates & & & & & & $\begin{array}{c}1736.6, \\
1233.6, \\
1041.3 \\
\text { (EtAc); } \\
2251.5 \\
\text { (ACN); } \\
1045.5 \\
\text { (DMSO); } \\
2928.5, \\
2872.7 \\
\text { (BuOH); } \\
1716.8 \\
\text { (BuON) }\end{array}$ & $\begin{array}{c}735.4 \\
\text { (DCM); } \\
2252.9 \\
(\mathrm{ACN}) ; \\
2961.2, \\
2936.6, \\
2875.8, \\
1051.5 \\
\text { (nPrOH); } \\
2966.9, \\
2936.6, \\
2874.4, \\
1046.2 \\
\text { (BuOH) } \\
1715.6, \\
1700.4, \\
1361.9, \\
1226.3 \\
\text { (ACT); } \\
3003.6, \\
747.2 \\
\text { (TCM) }\end{array}$ \\
\hline
\end{tabular}


Solid-state NMR analysis of FLU polymorphs. The assignment of the ${ }^{1} \mathrm{H}-{ }^{13} \mathrm{C} C P / M A S$ solid-state spectra was based on NQS and ${ }^{19} \mathrm{~F}-{ }^{13} \mathrm{C} \mathrm{CP} /$ MAS as well as CASTEP calculated isotropic chemical shifts (ESI Table S6) for FLU forms I, II, III, IV, and the FLU hydrate. As FLU forms I, III, and the hydrate have one FLU molecule in the asymmetric unit and FLU forms II and IV have two FLU molecules in the asymmetric unit, 13 and 26 peaks were expected in the solid-state NMR spectra, respectively. Albeit, the very similar local environment of triazole ring carbons resulted in overlapping of ${ }^{13} \mathrm{C}$ peaks in the 155 to 140 ppm spectral region. We were unable to assign the aromatic carbons $C 1$ and $C 5$ due to peaks broadening, hence carbons $\mathrm{C} 1$ and $\mathrm{C} 5$ were omitted in the RMSD calculations. The experimental and calculated (CASTEP) ${ }^{13} \mathrm{C}$ NMR peaks positions were in agreement as demonstrated by low RMSD values for the FLU polymorphs (below $2 \mathrm{ppm}$ ) and for the FLU hydrate (RMSD = $3.09 \mathrm{ppm}$ ). Despite the similar conformation of FLU molecules among forms I, II, III, and the hydrate (Figure 2B), distinct positions of NMR peaks were observed in the spectra. This is due to the differences in crystal packing, as well as the abundance of electronegative motifs (nitrogen, oxygen and fluorine atoms) positioned in close proximity to the ${ }^{13} \mathrm{C}$ atoms that alter the local environment of neighboring molecules in the polymorphs/hydrate. Furthermore, the presence of aromatic rings results in the occurrence of diamagnetic anisotropy phenomena observed as de-/shielding effects. For example, the position of carbon C4 can vary from $121.7 \mathrm{ppm}$ in form III to $126.3 \mathrm{ppm}$ in form I. The close proximity of carbon $\mathrm{C} 4$ to nitrogen $\mathrm{N} 5$ in the triazole ring and the angle between the triazole and difluorobenzene rings may have a deshielding effect observed as a C4 downfield peak shift from $121.7 \mathrm{ppm}$ in FLU form III to 125.9; 124.0 ppm in FLU form II and 126.3 ppm in FLU form I (C4 to N5 distance equals $3.358 \AA$ (FLU form III); $3.280 \AA$ and $3.269 \AA$ (FLU form II); $3.125 \AA$ (FLU form I)). A similar deshielding effect was observed for carbon $\mathrm{C} 6$ in FLU form II, positioned in close proximity to N3 of the adjacent molecule (3.298 $\AA$ ) in the plane to the triazole ring. This atom undergoes a 3.3 and 3.5 ppm downfield shift as compared to carbon C6 in FLU form I and FLU hydrate, respectively. Downfield shifts of carbons C2 and C2' in FLU form II (as compared to carbon C2 in forms I, III and the hydrate) can be explained by changes in electronic environment induced by proximity of oxygen atoms in the adjacent molecules $(3.26 ; 3.32$ $\AA$ ). Despite of almost identical conformation of both FLU molecules in FLU form IV the ${ }^{1} \mathrm{H}-{ }^{13} \mathrm{C} \mathrm{CP} / \mathrm{MAS}$ NMR spectra display distinct chemical shifts for carbons $\mathrm{C} 6, \mathrm{C} 3$ and $\mathrm{C} 2$ between both molecules. The largest difference of $4.2 \mathrm{ppm}$ between carbon C6 (102.3 ppm) and carbon C6' (106.5 ppm) can be explained by the spatial proximity of the N3' and F2' sites of a neighboring FLU to carbon $\mathrm{C6}^{\prime}$ ( $3.542 \AA$ and $3.860 \AA$, respectively). Similarly, the 2.0 ppm difference between carbons C2 (110.8 ppm) and C2' (112.8 ppm) may be explained by the close proximity of this site to fluorine F1 (3.362 $\AA$ ) of neighboring molecule. 
Table S6. Experimental and CASTEP calculated ${ }^{13} \mathrm{C}$ chemical shifts of FLU polymorphs and solvates.

\begin{tabular}{|c|c|c|c|c|c|c|c|c|c|c|c|c|c|c|}
\hline & $\begin{array}{l}\text { FLU I } \\
\text { exp. }\end{array}$ & $\begin{array}{l}\text { FLU I } \\
\text { calc. }\end{array}$ & $\begin{array}{l}\text { FLU II } \\
\text { exp. }\end{array}$ & $\begin{array}{l}\text { FLU II } \\
\text { calc. }\end{array}$ & $\begin{array}{l}\text { FLU III } \\
\text { exp. }\end{array}$ & $\begin{array}{l}\text { FLU III } \\
\text { calc. }\end{array}$ & $\begin{array}{l}\text { FLU IV } \\
\text { exp. }\end{array}$ & $\begin{array}{l}\text { FLU IV } \\
\text { calc. }\end{array}$ & $\begin{array}{c}\text { FLU HYD } \\
\text { exp. }\end{array}$ & $\begin{array}{c}\text { FLU HYD } \\
\text { calc. }\end{array}$ & $\begin{array}{c}\text { FLU DMSO } \\
\text { exp. }\end{array}$ & $\begin{array}{c}\text { FLU DMSO } \\
\text { calc. }\end{array}$ & $\begin{array}{c}\text { FLU DCM } \\
\text { exp. }\end{array}$ & $\begin{array}{c}\text { FLU DCM } \\
\text { calc. }\end{array}$ \\
\hline RMDS & \multicolumn{2}{|c|}{1.944} & \multicolumn{2}{|c|}{1.481} & \multicolumn{2}{|c|}{1.639} & \multicolumn{2}{|c|}{1.544} & \multicolumn{2}{|c|}{3.095} & \multicolumn{2}{|c|}{1.677} & \multicolumn{2}{|c|}{1.733} \\
\hline \multicolumn{15}{|l|}{$\mathrm{C} 1$} \\
\hline $\mathrm{C} 2$ & 112.1 & 109.93 & 113.7 & 113.21 & 111.2 & 108.95 & 110.8 & 110.22 & 110.8 & 107.55 & 112.5 & 110.96 & 113.8 & 113.74 \\
\hline $\mathrm{C} 3$ & 131.3 & 130.68 & 130.5 & 131.1 & 132.4 & 132.42 & 130.7 & 131.42 & 134.4 & 133.72 & 130.4 & 130.82 & 130.7 & 131.3 \\
\hline C4 & 126.3 & 125.66 & 124 & 124.33 & 121.7 & 120.79 & 122.2 & 123.42 & 122.9 & 121.79 & 126.5 & 127.54 & 123.7 & 124.03 \\
\hline $\mathrm{C} 6$ & 104.1 & 101.57 & 107.4 & 107.74 & 105.2 & 103.76 & 102.3 & 101.87 & 103.9 & 100.8 & 105.1 & 105.08 & 109.1 & 106.62 \\
\hline $\mathrm{C7}$ & 74.9 & 77.36 & 74.8 & 78.34 & 77.2 & 80.42 & 75.8 & 80.65 & 75.4 & 76.18 & 74.8 & 79.42 & 74.6 & 78.45 \\
\hline $\mathrm{C} 8$ & 57.2 & 54.81 & 56.2 & 54.72 & 58.8 & 57.58 & 57.6 & 57.44 & 58.0 & 53.19 & 57.2 & 56.6 & 56.3 & 54.17 \\
\hline C9 & 54.3 & 50.82 & 54.7 & 53.05 & 56.0 & 53.52 & 56.5 & 56.06 & 54.9 & 49.37 & 54.4 & 52.95 & 53.8 & 51.79 \\
\hline C10 & 147.4 & 146.39 & 146.5 & 145.3 & 145.4 & 144.42 & 143.8 & 142.18 & 147.6 & 145.76 & 147.3 & 146.72 & 146.7 & 145.95 \\
\hline C11 & 151.5 & 153.05 & 149.8 & 149.6 & 151.6 & 152.92 & 150.8 & 150.56 & 152 & 152.26 & 150.9 & 152.73 & 151.9 & 152.03 \\
\hline C13 & 150.3 & 149.86 & 151.6 & 150.84 & 151.6 & 151.65 & 150.8 & 150.94 & 148.3 & 148.69 & 150.9 & 149.19 & 152.3 & 152.48 \\
\hline \multicolumn{15}{|l|}{$\mathrm{C} 1^{\prime}$} \\
\hline $\mathrm{C} 2^{\prime}$ & & & 115.6 & 115.73 & & & 112.8 & 112.59 & & & & & & \\
\hline C3' & & & 130.5 & 131.26 & & & 129.4 & 129.64 & & & & & & \\
\hline $\mathrm{C} 4^{\prime}$ & & & 125.9 & 126.56 & & & 122.2 & 123.05 & & & & & & \\
\hline \multicolumn{15}{|l|}{ C5' } \\
\hline C6' & & & 106.5 & 105.62 & & & 106.5 & 106.83 & & & & & & \\
\hline$C 7^{\prime}$ & & & 74.8 & 78.18 & & & 75.8 & 79.93 & & & & & & \\
\hline C8' & & & 57.7 & 56.22 & & & 57.6 & 57.4 & & & & & & \\
\hline C9' & & & 54.7 & 52.8 & & & 57.6 & 57.77 & & & & & & \\
\hline $\mathrm{C} 10^{\prime}$ & & & 146.5 & 145.91 & & & 146.2 & 145.43 & & & & & & \\
\hline C11' & & & 153.6 & 154.63 & & & 152,1 & 151.94 & & & & & & \\
\hline C12' & & & 145.8 & 143.79 & & & 147.3 & 147.5 & & & & & & \\
\hline
\end{tabular}


Section S7. Stability of type II FLU solvates
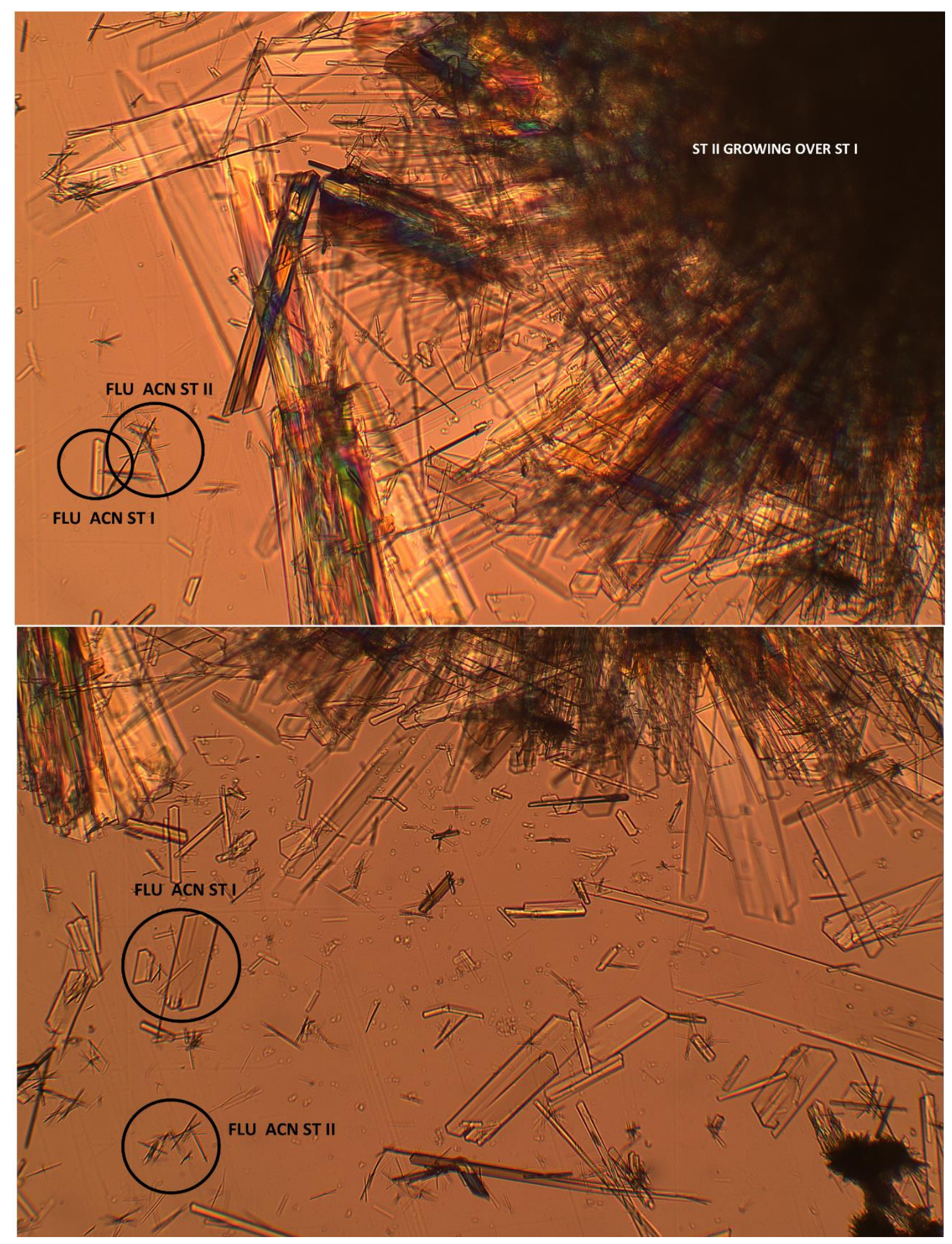

Figure S13. Microscopic images of FLU ACN STII and FLU ACN ST I crystals. 

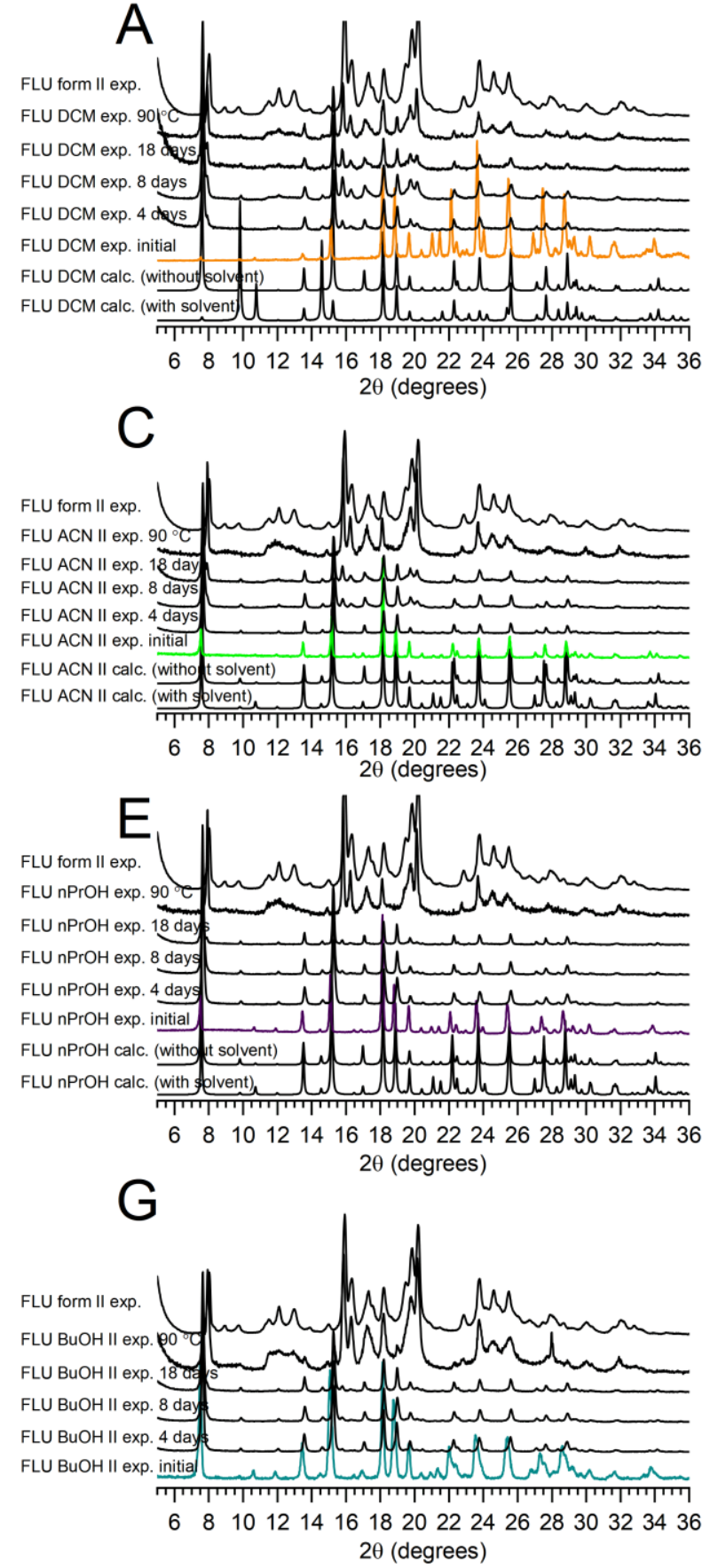
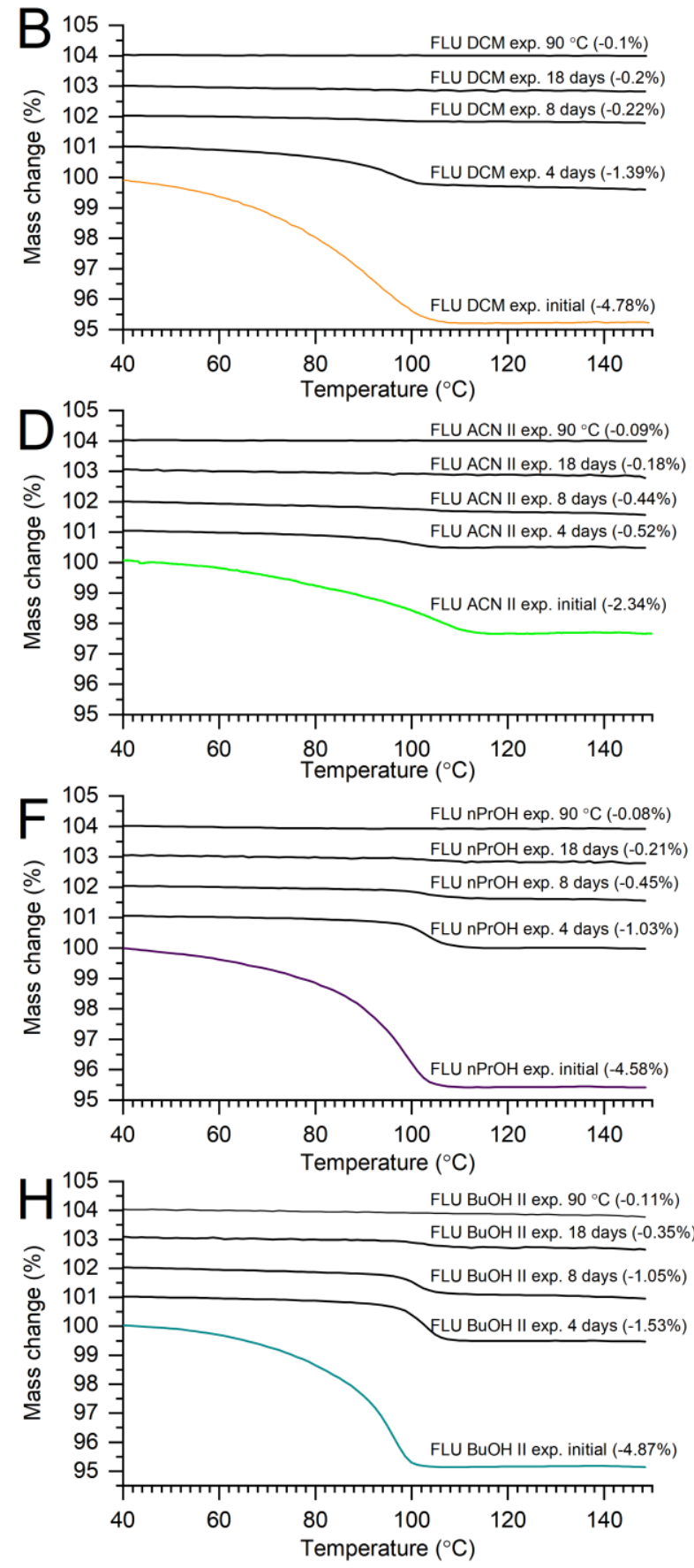

Figure S14. PXRD patterns (left) and TGA thermograms (righ) of vaccum dried type II FLU solvates after 4, 8, 18 days. 

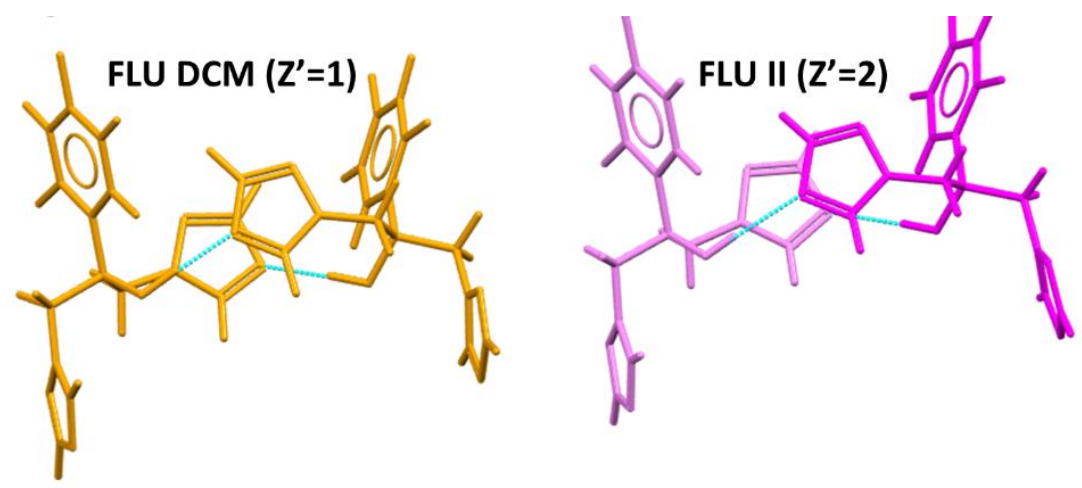

Figure S15. Comparison of FLU hydrogen bonded dimers of form II and DCM solvate. 
Section S8. ACT and TCM solvates
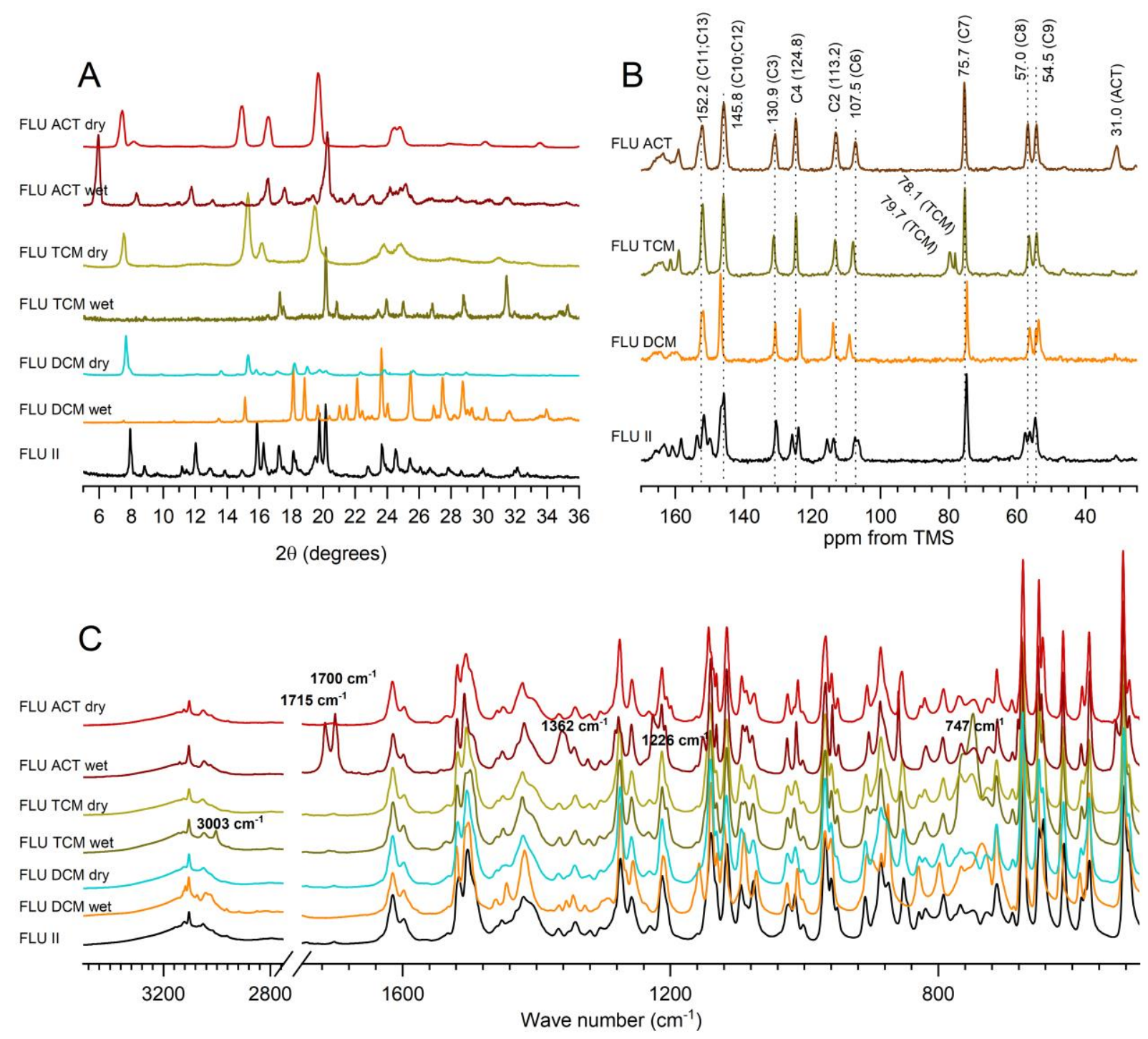

Figure S16. PXRD patterns (A), ${ }^{13} \mathrm{C}$ solid state NMR (B) and FTIR (C) spectra of FLU solvates with ACT and TCM. 


\section{Section S9. Type II fluconazole solvates - structure solution}

The PXRD patterns were recorded using an X'Pert PRO diffractometer (PANalytical, Almelo, NL), equipped with a $\theta / \theta$ coupled goniometer in transmission geometry, programmable XYZ stage with well plate holder, $\mathrm{Cu}-\mathrm{K \alpha}_{1,2}$ radiation source and a solid state PIXcel detector. The patterns were recorded at a tube voltage of $40 \mathrm{kV}$ and tube current of $40 \mathrm{~mA}$, applying a step size of $2 \theta=0.013^{\circ}$ with $4000 \mathrm{~s}$ per step in the $2 \theta$ range between $2^{\circ}$ and $60^{\circ}$.

The diffraction patterns of FLU DCM, FLU ACN II, and FLU nPrOH indexed to a tetragonal unit cell using the first twenty peaks with DICVOLO4 and the space group was determined to be $P \overline{4} 2_{1} c$ based on a statistical assessment of systematic absences, ${ }^{16}$ as implemented in the DASH structure solution package. ${ }^{17}$ From the cell volume it was derived that there is one FLU molecule in the asymmetric unit and from thermogravimetric analysis that there are 0.25 moles of solvent per mole FLU. The data were background subtracted and Pawley refinement ${ }^{18}$ was used to extract the intensities and their correlations. Simulated annealing was used to optimize the three solvate models against the diffraction data set in direct space. The internal coordinate (Z-matrix) descriptions were derived from the PBE0/6$31 \mathrm{G}(\mathrm{d}, \mathrm{p})$ gas phase global conformational minima, with $\mathrm{O}-\mathrm{H}$ distances normalized to $0.9 \AA$ and $\mathrm{C}-\mathrm{H}$ distances to $0.95 \AA$. Each of the structures was solved using 200 simulated annealing runs of $5 \times 10^{7}$ moves per run in DASH. Each FLU molecule was allowed 6 external and 5 internal degrees of freedom and DCM and ACN each 6 external degrees of freedom and nPrOH 7 degrees of freedom ( 6 external, 1 internal). In the case of the solvent molecules, the $\mathrm{H}$ atoms were omitted and the occupancies first fixed to 0.25 each. In case of FLU nPrOH the best solutions returned a $\chi^{2}$ ratio of ca. 2.42 (profile $\chi^{2}$ / pawley $\chi^{2}$ ). Reducing the occupancy rate of the FLU DCM and FLU ACN II in the simulated annealing runs resulted in better (profile $\chi^{2} /$ pawley $\chi^{2}$ ) ratios, 5.30 and 4.27 for FLU DCM and FLU ACN II, respectively, using occupancy of 0.025 .

The best solutions were then subjected to full and fixed cell (atomic positions optimized) CASTEP ${ }^{19}$ optimizations. The DFT-D calculations were carried out with the CASTEP plane wave code using the Perdew-Burke-Ernzerhof (PBE) generalized gradient approximation (GGA) exchange-correlation density functional and ultrasoft pseudopotentials with the addition of the Tkatchenko and Scheffler (TS) semi-empirical dispersion corrections. ${ }^{20}$ Brillouin zone integrations were performed on a symmetrized Monkhorst-Pack k-point grid with the number of $k$-points chosen to provide a maximum spacing of $0.07 \AA^{-1}$ and a basis set cut-off of $780 \mathrm{eV}$. The self-consistent field convergence on total energy was set to $1 \times 10^{-5} \mathrm{eV}$ per atom. Energy minimizations were performed using the BroydenFletcher-Goldfarb-Shanno optimisation scheme within the space group constraints. The optimizations were considered complete when energies were converged to better than $2 \times 10^{-5} \mathrm{eV}$ per atom, atomic displacements converged to $1 \times 10^{-3} \AA$, maximum forces to $5 \times 10^{-2} \mathrm{eV} \AA^{-1}$, and maximum stresses were converged to $1 \times 10^{-1} \mathrm{GPa}$. The different optimisations were then compared to the results from the simulated annealing and confirmed isostructurality.

The PBE-TS structures (fixed cell parameters) with with $\mathrm{O}-\mathrm{H}$ distances normalized to $0.9 \AA$ and $\mathrm{C}-\mathrm{H}$ distances to $0.95 \AA$ were then used as the starting point for rigid body Rietveld refinements ${ }^{21}$ in TOPAS V7.12. ${ }^{22}$ The final refinements included a total of 48 parameters ( 26 profile, 2 cell, 1 scale, 1 isotropic temperature factor, 6 position and 6 rotation, 5 preferred orientation, 1 solvent occupancy) yielding a final $R_{w p}=5.13 \%$ for FLU nPrOH, with a refined solvent occupancy of $0.219(2)$. In case of FLU DCM and FLU ACN II the solvent occupancy refined to 0.03(3) and 0.03(2), respectively, and therefore the Rietveld refinements were repeated without solvent molecules. The final refinements included then a total of 41 parameters ( 26 profile, 2 cell, 1 scale, 1 isotropic temperature factor, 3 position, 3 rotation, 
5 preferred orientation) and yielded final $R_{w p}$ values of $6.25 \%$ and $6.09 \%$ for FLU DCM and FLU ACN II, respectively (Figure S16).
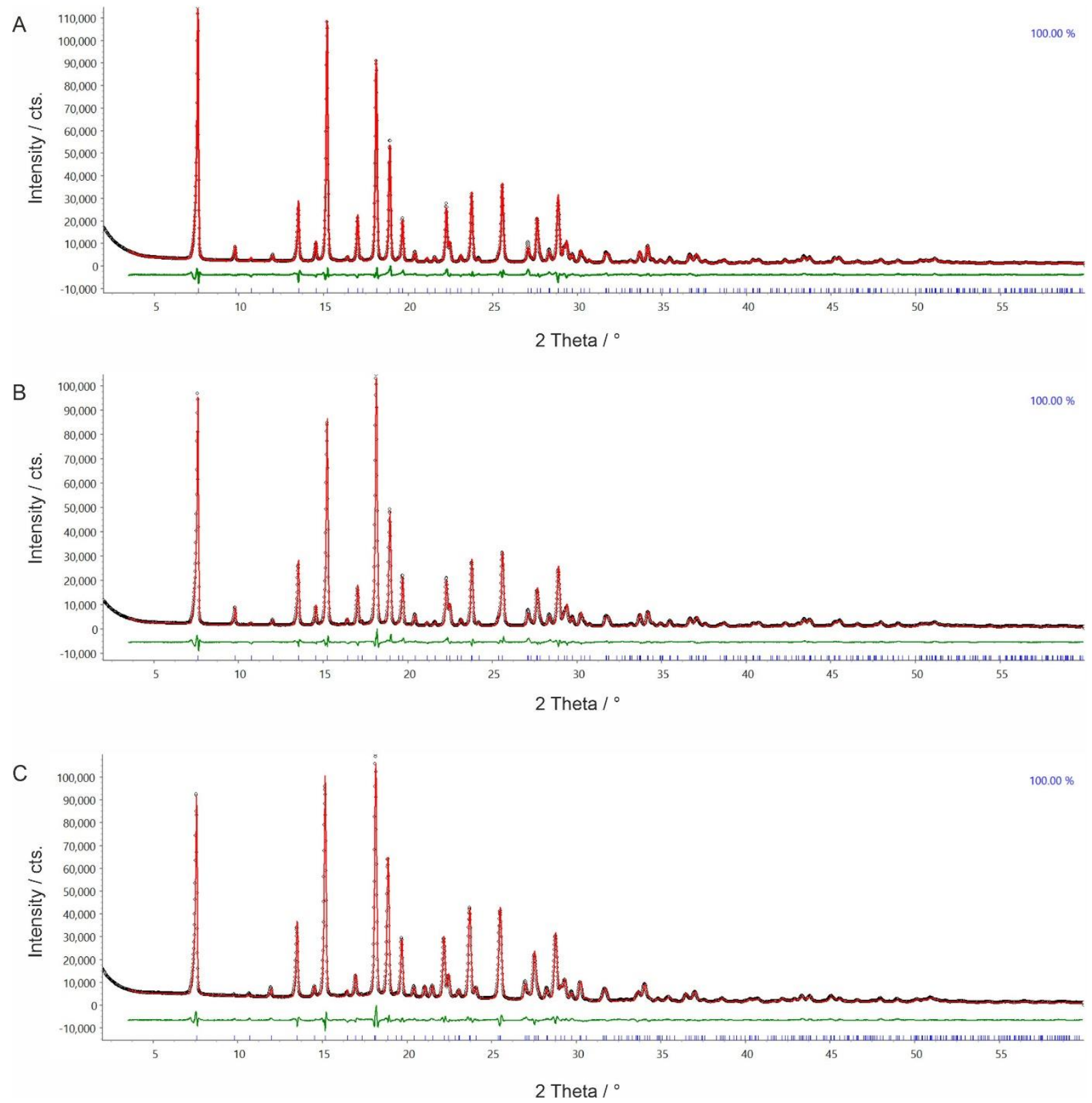

Figure S17. Final observed (points), calculated (red line) and difference (green) profiles for the Rietveld refinement of (A) FLU DCM, (B) FLU ACN II, and (C) FLU nPrOH. 


\section{References}

1. Lo, J. B., Mackay, G. G. \& Puz, M. J. UK Patent Application GB 2270521A. (1994).

2. Gu, X. J. \& Jlang, W. Characterization of Polymorphic Forms of Fluconazole Using Fourier Transform Raman Spectroscopy. J. Pharm. Sci. 84, 1438-1441 (1995).

3. Dash, A. K. \& Elmquist, W. F. Fluconazole. in vol. 21 67-113 (2001).

4. Desai, S. R., Shaikh, M. M. \& Dharwadkar, S. R. Thermoanalytical study of polymorphic transformation in fluconazole drug. Thermochim. Acta 399, 81-89 (2002).

5. Alkhamis, K. A., Obaidat, A. A. \& Nuseirat, A. F. Solid-State Characterization of Fluconazole. Pharm. Dev. Technol. 7, 491-503 (2002).

6. Caira, M. R., Alkhamis, K. A. \& Obaidat, R. M. Preparation and Crystal Characterization of a Polymorph, a Monohydrate, and an Ethyl Acetate Solvate of the Antifungal Fluconazole. J. Pharm. Sci. 93, 601-611 (2004).

7. Kreidl, J. et al. Patent WO2002076955A1. (2002).

8. $\quad$ Kreidl, J. et al. U.S. Patent, US 007094904 B2. (2006) doi:10.1145/634067.634234.

9. Karanam, M., Dev, S. \& Choudhury, A. R. New polymorphs of fluconazole: Results from cocrystallization experiments. Cryst. Growth Des. 12, 240-252 (2012).

10. Basford, P. A. et al. Impact of Crystal Structure and Molecular Conformation on the Hydration Kinetics of Fluconazole. Cryst. Growth Des. 19, 7193-7205 (2019).

11. Abraham, M. H. Scales of solute hydrogen-bonding: their construction and application to physicochemical and biochemical processes. Chem. Soc. Rev. 22, 73 (1993).

12. Bell, G., Janssen, A. E. \& \& Halling, P. J. Water activity fails to predict critical hydration level for enzyme activity in polar organic solvents: Interconversion of water concentrations and activities. Enzyme Microb. Technol. 20, 471477 (1997).

13. Zhu, H., Yuen, C. \& Grant, D. J. W. Influence of water activity in organic solvent + water mixtures on the nature of the crystallizing drug phase. 1. Theophylline. Int. J. Pharm. 135, 151-160 (1996).

14. Blandamer, M. J., Engberts, J. B. F. N., Gleeson, P. T. \& Reis, J. C. R. Activity of water in aqueous systems; A frequently neglected property. Chem. Soc. Rev. 34, 440 (2005).

15. Allan, M. \& Mauer, L. J. Dataset of water activity measurements of alcohol:water solutions using a Tunable Diode Laser. Data Br. 12, 364-369 (2017).

16. Markvardsen, A. J., David, W. I. F., Johnson, J. C. \& Shankland, K. A probabilistic approach to space-group determination from powder diffraction data. Acta Crystallogr. Sect. A Found. Crystallogr. 57, 47-54 (2001).

17. David, W. I. F. et al. DASH : a program for crystal structure determination from powder diffraction data. J. Appl. Crystallogr. 39, 910-915 (2006).

18. Pawley, G. S. Unit-cell refinement from powder diffraction scans. J. Appl. Crystallogr. 14, 357-361 (1981).

19. Clark, S. J. et al. First principles methods using CASTEP. Zeitschrift für Krist. - Cryst. Mater. 220, 567-570 (2005).

20. Tkatchenko, A. \& Scheffler, M. Accurate Molecular Van Der Waals Interactions from Ground-State Electron Density and Free-Atom Reference Data. Phys. Rev. Lett. 102, 073005 (2009).

21. Rietveld, H. M. A profile refinement method for nuclear and magnetic structures. J. Appl. Crystallogr. 2, 65-71 (1969).

22. Coelho, A. Topas Academic Coelho Software. (2016). 